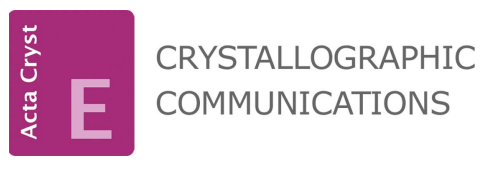

ISSN 2056-9890
Received 27 January 2016

Accepted 15 February 2016

Edited by S. Parkin, University of Kentucky, USA

Keywords: crystal structure; 1,8-diazabicyclo[5.4.0] undec-7-ene; BDU; benzoate salts; hydrogen bonding.

CCDC references: $1453494 ; 1453493$; 1453492

Supporting information: this article has supporting information at journals.iucr.org/e

\section{Crystal structures of three anhydrous salts of the Lewis base 1,8-diazabicyclo[5.4.0]undec-7-ene (DBU) with the ring-substituted benzoic acid analogues 4-aminobenzoic acid, 3,5-dinitrobenzoic acid and 3,5-dinitrosalicylic acid}

\author{
Graham Smith $^{\mathrm{a} *}$ and Daniel E. Lynch ${ }^{\mathrm{b}}$
}

${ }^{a}$ Science and Engineering Faculty, Queensland University of Technology, GPO Box 2434, Brisbane, Queensland 4001, Australia, and ${ }^{\mathbf{b}}$ Exilica Ltd, The Technocentre, Puma Way, Coventry CV1 2TT, England. *Correspondence e-mail: g.smith@qut.edu.au

The anhydrous salts of the Lewis base 1,8-diazabicyclo[5.4.0]undec-7-ene (DBU) with 4-aminobenzoic acid [1-aza-8-azoniabicyclo[5.4.0]undec-7-ene 4aminobenzoate, $\mathrm{C}_{9} \mathrm{H}_{17} \mathrm{~N}_{2}{ }^{+} \cdot \mathrm{C}_{7} \mathrm{H}_{6} \mathrm{NO}_{2}^{-}$(I)], 3,5-dinitrobenzoic acid [1-aza-8azoniabicyclo[5.4.0]undec-7-ene 3,5-dinitrobenzoate, $\mathrm{C}_{9} \mathrm{H}_{17} \mathrm{~N}_{2}{ }^{+} \cdot \mathrm{C}_{7} \mathrm{H}_{3} \mathrm{~N}_{2} \mathrm{O}_{6}{ }^{-}$, (II)] and 3,5-dinitrosalicylic acid (DNSA) [1-aza-8-azoniabicyclo[5.4.0]undec7-ene 2-hydroxy-3,5-dinitrobenzoate, $\mathrm{C}_{9} \mathrm{H}_{17} \mathrm{~N}_{2}{ }^{+} \cdot \mathrm{C}_{7} \mathrm{H}_{3} \mathrm{~N}_{2} \mathrm{O}_{7}{ }^{-}$, (III)] have been determined and their hydrogen-bonded structures are described. In both (II) and (III), the DBU cations have a common disorder in three of the $\mathrm{C}$ atoms of the six-membered ring moieties [site-occupancy factors $(\mathrm{SOF})=0.735(3) /$ 0.265 (3) and 0.686 (4)/0.314 (4), respectively], while in (III), there is additional rotational disorder in the DNSA anion, giving two sites ( $\mathrm{SOF}=0.72 / 0.28$, values fixed) for the phenol group. In the crystals of (I) and (III), the cation-anion pairs are linked through a primary $\mathrm{N}-\mathrm{H} \cdots \mathrm{O}_{\text {carboxyl }}$ hydrogen bond $[2.665$ (2) and 2.869 (3) $\AA$, respectively]. In (II), the ion pairs are linked through an asymmetric three-centre $R_{1}^{2}(4), \mathrm{N}-\mathrm{H} \cdots \mathrm{O}, \mathrm{O}^{\prime}$ chelate association. In (I), structure extension is through amine $\mathrm{N}-\mathrm{H} \cdots \mathrm{O}_{\text {carboxyl }}$ hydrogen bonds between the PABA anions, giving a three-dimensional structure. The crystal structures of (II) and (III) are very similar, the cation-anion pairs being associated only through weak $\mathrm{C}-$ $\mathrm{H}$.. O hydrogen bonds, giving in both overall two-dimensional layered structures lying parallel to (001). No $\pi-\pi$ ring associations are present in any of the structures.

\section{Chemical context and database survey}

The Lewis base 1,8-diazabicyclo[5.4.0]undec-7-ene (DBU) is an alkaloid isolated from the sponge Niphates digitalis (Regalado et al., 2010) but is commonly synthesized. It finds use as a curing agent for epoxy resins, as a catalyst in organic syntheses, and as a counter-cation in metal complex chemistry, e.g. with the pentabromo(triphenylphosphane)platinum(IV) monoanion (Motevalli et al., 1989). It has also found use in binding organic liquids (BOLs), which usually comprise a mixture of amidines or guanidine and alcohol, and are used to reversibly capture and release gases such as $\mathrm{CO}_{2}, \mathrm{CS}_{2}, \mathrm{SO}_{2}$ or COS (Shannon et al., 2015; Pérez et al., 2004; Heldebrant et al., 2009). The structure of one of these formed from the absorption of $\mathrm{CO}_{2}$ is the bicarbonate (Pérez et al., 2004).

As a very strong base $\left(\mathrm{p} K_{\mathrm{a}} c a 14\right)$, protonation of the $\mathrm{N} 8$ group of the six-membered hetero-ring of DBU is readily achieved and results in the formation of salts with carboxylic 
acids and phenols. The Cambridge Structural Database (2015 version) (Groom \& Allen, 2014) contains 35 examples of organic salts of DBU, among them the benzyl dithiocarbonate (Heldebrant et al., 2009) and the phenolate from 2,6-di(tertbutyl)-4-nitrophenol (Lynch \& McClenaghan, 2003). However, of the total there are surprisingly few carboxylate salts, e.g. with Kemp's triacid (1,3,5-trimethylcyclohexane1,3,5-tricarboxylic acid) (a monoanionic acetonitrile salt) (Huczyński et al., 2008) and the dianionic salt of the tetra(3carboxyphenyl)-substituted porphyrin (Lipstman \& Goldberg, 2013).

No reported crystal structures of salts with simple substituted benzoic acids are found, so in order to examine the hydrogen-bonding in crystals of the DBU salts with some common ring-substituted benzoic acids, a number of these were prepared. Suitable crystals were obtained with 4aminobenzoic acid (PABA), (3,5-dinitrobenzoic acid (DNBA) and (3,5-dinitrosalicylic acid (DNSA), giving the anhydrous salts, $\mathrm{C}_{9} \mathrm{H}_{17} \mathrm{~N}_{2}{ }^{+} \mathrm{C}_{7} \mathrm{H}_{6} \mathrm{NO}_{2}{ }^{-}$(I), $\mathrm{C}_{9} \mathrm{H}_{17} \mathrm{~N}_{2}{ }^{+} \mathrm{C}_{7} \mathrm{H}_{3} \mathrm{~N}_{2} \mathrm{O}_{6}{ }^{-}$(II) and $\mathrm{C}_{9} \mathrm{H}_{17} \mathrm{~N}_{2}^{+} \mathrm{C}_{7} \mathrm{H}_{3} \mathrm{~N}_{2} \mathrm{O}_{7}^{-}$(III), respectively and their structures and hydrogen-bonding modes are reported herein.<smiles>C1CCC2=[NH+]CCCN2CC1</smiles>

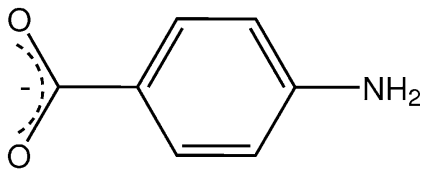

(I)<smiles>C1CCC2=[NH+]CCCN2CC1</smiles>

(II)

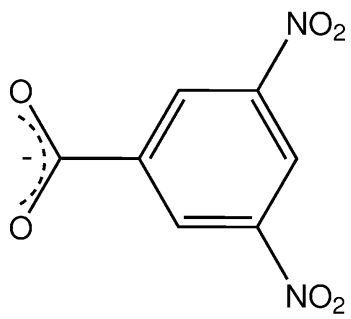<smiles>C1CCC2=[NH+]CCCN2CC1</smiles>

(III)

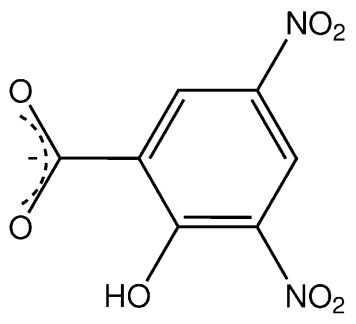

\section{Structural commentary}

The asymmetric units of (I)-(III) comprise a BDU cation $(A)$ and a 4-aminobenzoate anion (B), (I) (Fig. 1), a 3,5-dinitrobenzoate anion (B), (II) (Fig. 2), and a 3,5-dinitrosalicylate anion (B), (III) (Fig. 3). The cation-anion pairs in (I) and (III)

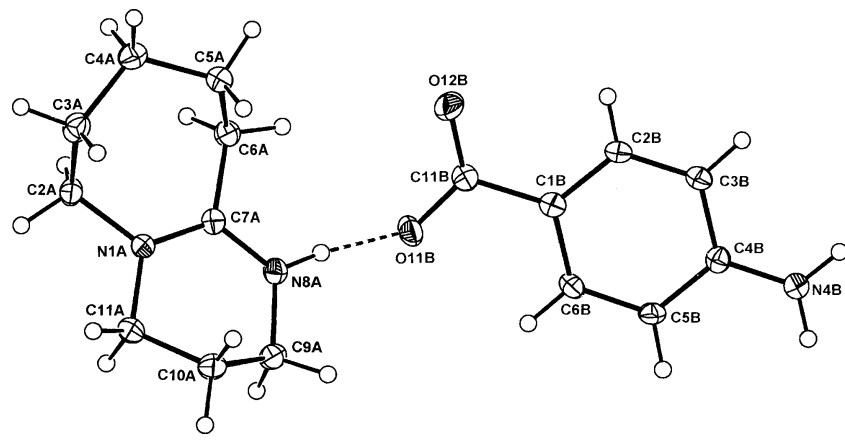

Figure 1

The atom-numbering scheme and the molecular conformation of the DBU cation $(A)$ and the PABA anion $(B)$ in (I) with displacement ellipsoids drawn at the $40 \%$ probability level. The cation-anion hydrogen bond is shown as a dashed line.

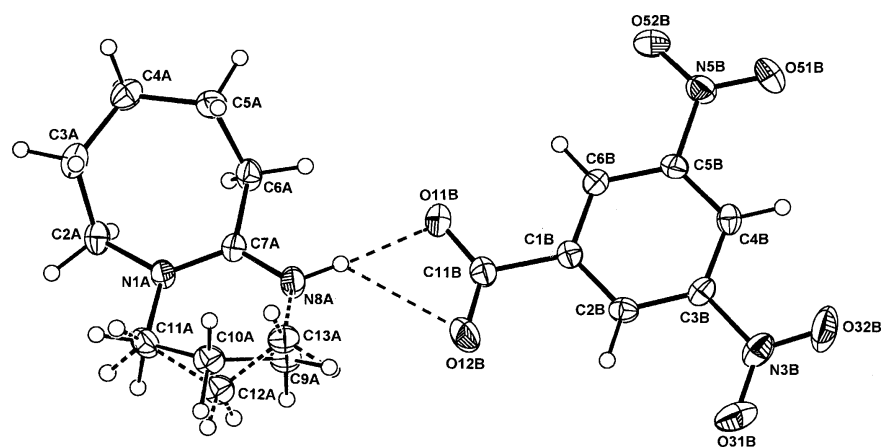

Figure 2

The atom-numbering scheme and the molecular conformation of the DBU cation $(A)$ and the DNBA anion $(B)$ in (II) with displacement ellipsoids drawn at the $40 \%$ probability level. The bonds in the minor disordered section of the six-membered ring of the cation and the cationanion hydrogen bonds are shown as dashed lines.

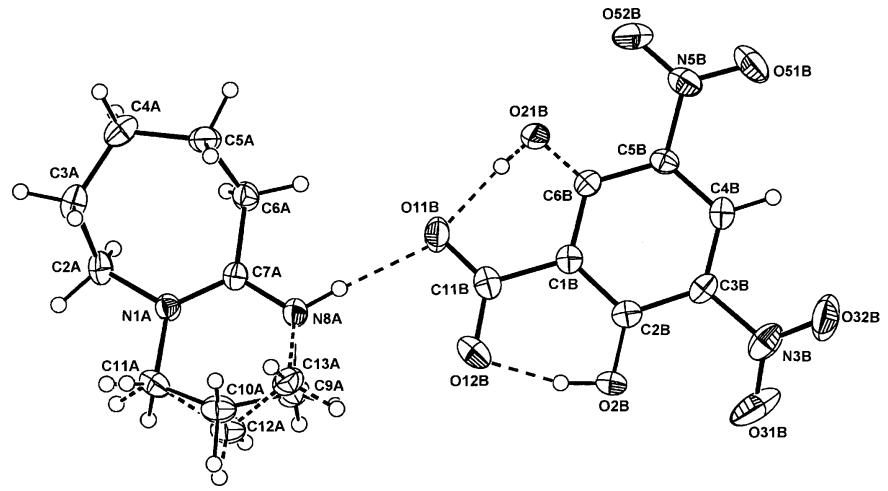

Figure 3

The atom-numbering scheme and the molecular conformation of the DBU cation $(A)$ and the DNSA anion $(B)$ in (III) with displacement ellipsoids drawn at the $40 \%$ probability level. The bonds in the minor disordered section of the six-membered ring of the cation are shown as dashed lines.

are linked through a primary $\mathrm{N} 8 A-\mathrm{H} \cdots \mathrm{O}_{\text {carboxyl }}$ hydrogen bond [2.665 (2) and 2.871 (3) A, respectively; Tables 1 and 3]. In (II), the ion pairs are linked through an asymmetric threecentre $R_{1}^{2}(4), \mathrm{N} 8 A-\mathrm{H} \cdots O, O^{\prime}$ chelate association [2.777 (2), 3.117 (2) $\AA$; Table 2]. With (III), the corresponding longer contact with the second carboxyl O12B atom is 3.222 (3) $\AA$ (Fig. 3). 


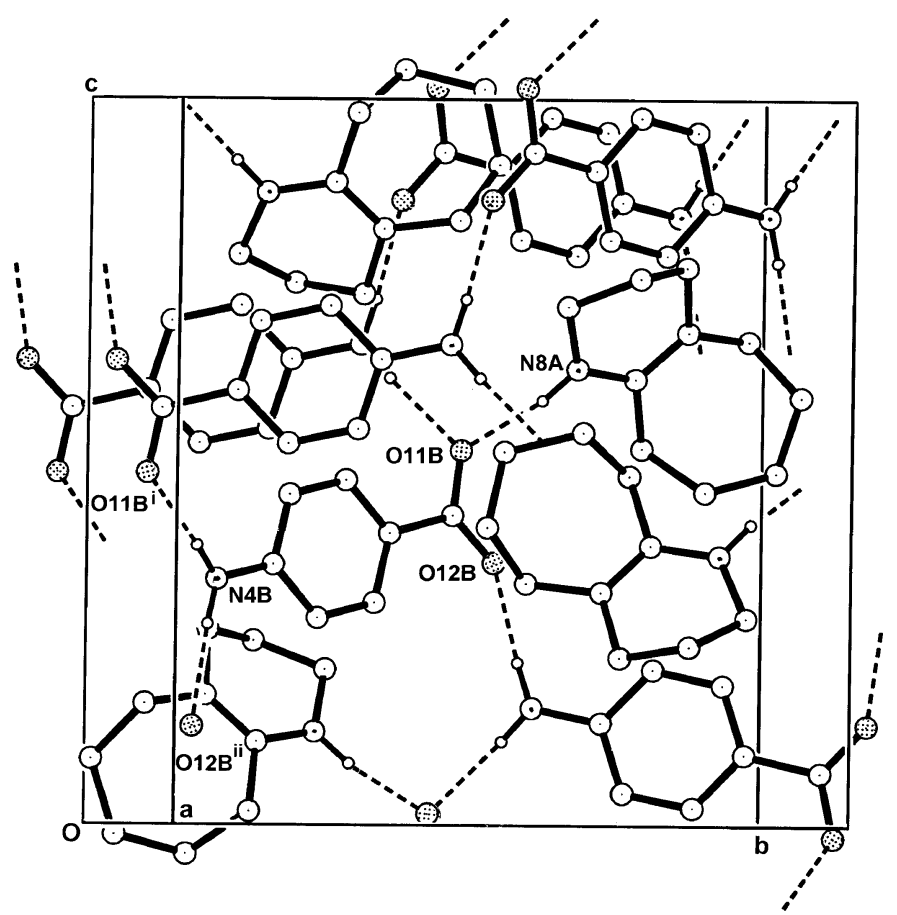

Figure 4

The three-dimensional hydrogen-bonded framework structure of (I) viewed approximately along $a$. For symmetry codes, see Table 1 .

With the structures of (II) and (III), there is disorder in the six-membered ring system involving atoms $\mathrm{C} 9 A$ and $\mathrm{C} 10 A$ (with alternative minor occupancy sites $\mathrm{C} 12 A$ and $\mathrm{C} 13 A$ ), giving similar site occupancy factors [SOF 0.735 (3)/0.265 (3) and 0.686 (4)/0.314 (4) for (II) and (III), respectively]. This feature is found in three other structures among the CSD set: the previously mentioned 2,6-di(tert-butyl)-4-nitrophenolate (SOF 0.60/0.40) (Lynch \& McClenaghan, 2003); in the

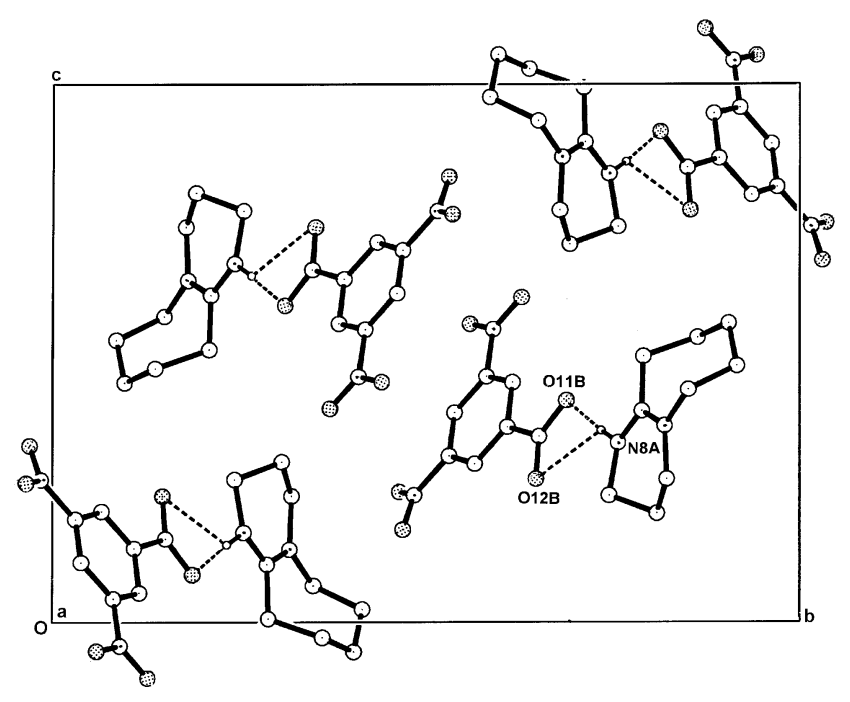

Figure 5

The packing of the hydrogen-bonded cation-anion pairs in the unit cell of (II), viewed along $a$. The minor-component disordered atoms and the non-associative $\mathrm{H}$ atoms have been omitted.
Table 1

Hydrogen-bond geometry $\left(\AA,^{\circ}\right)$ for (I).

\begin{tabular}{lllll}
\hline$D-\mathrm{H} \cdots A$ & $D-\mathrm{H}$ & $\mathrm{H} \cdots A$ & $D \cdots A$ & $D-\mathrm{H} \cdots A$ \\
\hline $\mathrm{N} 8 A-\mathrm{H} 8 A \cdots \mathrm{O} 11 B$ & $0.89(2)$ & $1.78(2)$ & $2.665(2)$ & $170(2)$ \\
$\mathrm{N} 4 B-\mathrm{H} 41 B \cdots \mathrm{O} 11 B^{\mathrm{i}}$ & $0.89(2)$ & $2.05(2)$ & $2.939(2)$ & $176(2)$ \\
$\mathrm{N} 4 B-\mathrm{H} 42 B \cdots \mathrm{O} 12 B^{\mathrm{ii}}$ & $0.92(2)$ & $1.98(2)$ & $2.891(2)$ & $176(2)$ \\
\hline
\end{tabular}

Symmetry codes: (i) $x+\frac{1}{2},-y+\frac{1}{2},-z+1$; (ii) $-x+1, y-\frac{1}{2},-z+\frac{1}{2}$.

Table 2

Hydrogen-bond geometry $\left(\AA{ }^{\circ}\right)$ for (II).

\begin{tabular}{lllll}
\hline$D-\mathrm{H} \cdots A$ & $D-\mathrm{H}$ & $\mathrm{H} \cdots A$ & $D \cdots A$ & $D-\mathrm{H} \cdots A$ \\
\hline $\mathrm{N} 8 A-\mathrm{H} 8 A \cdots \mathrm{O} 11 B$ & $0.90(2)$ & $1.88(2)$ & $2.777(2)$ & $177(2)$ \\
$\mathrm{N} 8 A-\mathrm{H} 8 A \cdots \mathrm{O} 12 B$ & $0.90(2)$ & $2.53(2)$ & $3.117(2)$ & $124(1)$ \\
$\mathrm{C} 10 A-\mathrm{H} 11 A \cdots \mathrm{O} 32 B^{\mathrm{i}}$ & 0.99 & 2.44 & $3.247(3)$ & 138 \\
$\mathrm{C} 2 A-\mathrm{H} 21 A \cdots \mathrm{O} 31 B^{\mathrm{ii}}$ & 0.99 & 2.56 & $3.309(2)$ & 133 \\
$\mathrm{C} 6 A-\mathrm{H} 62 A \cdots \mathrm{O} 11 B$ & 0.99 & 2.60 & $3.438(2)$ & 143 \\
\hline
\end{tabular}

Symmetry codes: (i) $-x-\frac{1}{2}, y+\frac{1}{2},-z+\frac{1}{2}$; (ii) $-x+\frac{1}{2}, y+\frac{1}{2},-z+\frac{1}{2}$.

Table 3

Hydrogen-bond geometry $\left(\AA,^{\circ}\right)$ for (III).

\begin{tabular}{lllll}
\hline$D-\mathrm{H} \cdots A$ & $D-\mathrm{H}$ & $\mathrm{H} \cdots A$ & $D \cdots A$ & $D-\mathrm{H} \cdots A$ \\
\hline $\mathrm{N} 8 A-\mathrm{H} 8 A \cdots \mathrm{O} 11 B$ & $0.88(2)$ & $1.99(2)$ & $2.871(3)$ & $176(2)$ \\
$\mathrm{O} 2 B-\mathrm{H} 2 B \cdots \mathrm{O} 12 B$ & 0.84 & 1.72 & $2.473(3)$ & 149 \\
$\mathrm{C} 10 A-\mathrm{H} 11 A \cdots \mathrm{O} 32 B^{\mathrm{i}}$ & 0.99 & 2.45 & $3.251(5)$ & 138 \\
$\mathrm{C} 2 A-\mathrm{H} 21 A \cdots \mathrm{O} 31 B^{\text {ii }}$ & 0.99 & 2.48 & $3.281(3)$ & 138 \\
\hline
\end{tabular}

Symmetry codes: (i) $-x+\frac{3}{2}, y+\frac{1}{2},-z+\frac{3}{2}$; (ii) $-x+\frac{1}{2}, y+\frac{1}{2},-z+\frac{3}{2}$.

8-bromoguanosine 8-bromoguanoside adduct salt ( $\mathrm{SOF}=$ 0.63/0.37) (Saftić et al., 2012) and in the counter-cation of a bromocarbyne Mo complex $(\mathrm{SOF}=0.83 / 0.17)$ (Cordiner et al., 2008).

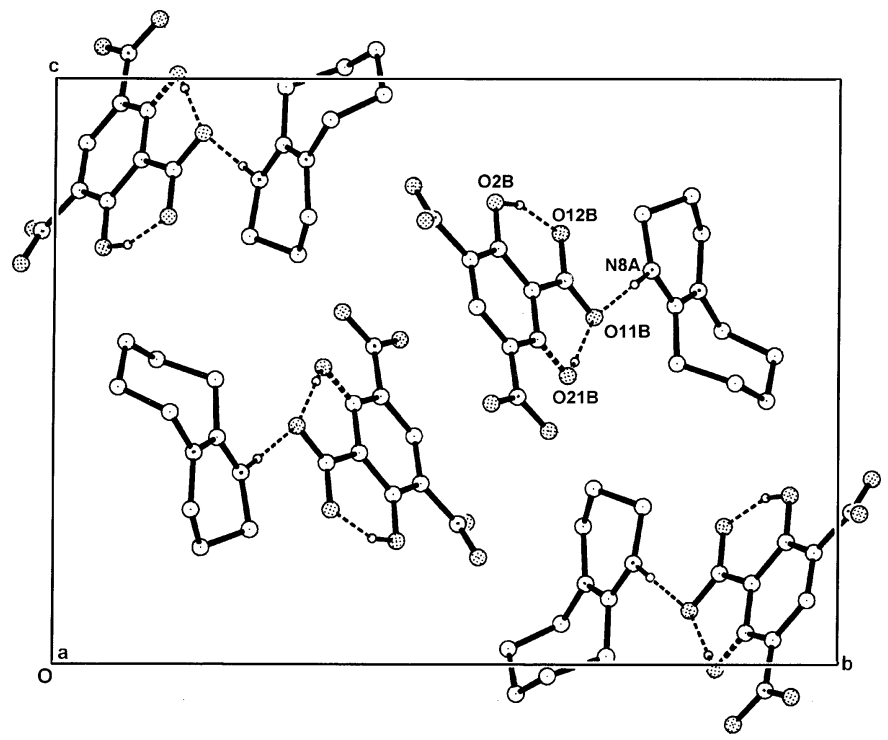

Figure 6

The packing of the hydrogen-bonded cation-anion pairs in the unit cell of (III), viewed along $a$. The minor-component disordered atoms and the non-associative $\mathrm{H}$ atoms have been omitted. 
Table 4

Experimental details.

(I)

Crystal data

Chemical formula

$M_{\mathrm{r}}$

Crystal system, space group

Temperature (K)

$a, b, c(\AA)$

$\alpha, \beta, \gamma\left(^{\circ}\right)$

$V\left(\AA^{3}\right)$

$Z$

Radiation type

$\mu\left(\mathrm{mm}^{-1}\right)$

Crystal size (mm)

Data collection

Diffractometer

Absorption correction

$T_{\min }, T_{\max }$

No. of measured, independent and

observed $[I>2 \sigma(I)]$ reflections

$R_{\text {int }}$

$(\sin \theta / \lambda)_{\max }\left(\AA^{-1}\right)$

$\mathrm{C}_{9} \mathrm{H}_{17} \mathrm{~N}_{2}{ }^{+} \cdot \mathrm{C}_{7} \mathrm{H}_{6} \mathrm{NO}_{2}{ }^{-}$

289.37

Orthorhombic, $P 2_{1} 2_{1} 2_{1}$

200

8.0986 (4), 12.9213 (6), 13.7344 (7)

90, 90, 90

$1437.23(12)$

4

Mo $K \alpha$

0.09

$0.40 \times 0.26 \times 0.24$

Oxford Diffraction Gemini-S

CCD-detector

Multi-scan (CrysAlis PRO;

Agilent, 2014)

$0.93,0.99$

$7372,3324,2847$

0.031

0.687

$0.044,0.098,1.07$

3324

199

3

$\mathrm{H}$ atoms treated by a mixture of independent and constrained refinement

$0.20,-0.25$

(II)

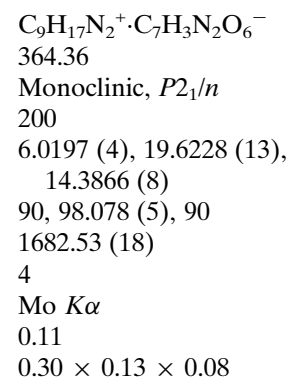

$\mathrm{C}_{9} \mathrm{H}_{17} \mathrm{~N}_{2}{ }^{+} \cdot \mathrm{C}_{7} \mathrm{H}_{3} \mathrm{~N}_{2} \mathrm{O}_{6}{ }^{-}$

364.36

Monoclinic, $P 2_{1} / n$

200

6.0197 (4), 19.6228 (13),

$14.3866(8)$

90, $98.078(5), 90$

1682.53 (18)

Mo $K \alpha$

0.11

$0.30 \times 0.13 \times 0.08$

Oxford Diffraction Gemini-S CCD-detector

Multi-scan (CrysAlis PRO;

Agilent, 2014)

$0.90,0.99$

$7082,3311,2561$

0.024

0.617

$0.045,0.109,1.02$
3311
245
3
$\mathrm{H}$ atoms treated by a mixture of
$\quad$ independent and constrained
$\quad$ refinement
$\quad 0.18,-0.22$

$0.18,-0.22$
(III)

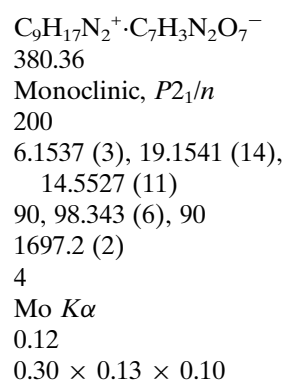

Oxford Diffraction Gemini-S CCD-detector

Multi-scan (CrysAlis PRO; Agilent, 2014)

$0.920,0.990$

$7800,3339,2347$

0.034

0.617

$0.058,0.123,1.03$
3339
263
3
$\mathrm{H}$ atoms treated by a mixture of
$\quad$ independent and constrained
$\quad$ refinement
$0.29,-0.29$

$0.29,-0.29$

$\Delta \rho_{\max }, \Delta \rho_{\min }\left(\mathrm{e} \AA^{-3}\right)$

Computer programs: CrysAlis PRO (Agilent, 2014), SIR92 (Altomare et al., 1993), SHELXL97 (Sheldrick, 2008) within WinGX (Farrugia, 2012) and PLATON (Spek, 2009).

With the PABA anion in (I), the carboxylate group is essentially coplanar with the benzene ring [torsion angle $\mathrm{C} 2 B-\mathrm{C} 1 B-\mathrm{C} 11 B-\mathrm{O} 11 B=179.25(15)^{\circ}$, a feature similar to those found in the parent acid (Gracin \& Fischer, 2005) and its co-crystals, e.g. with 4-nitrobenzoic acid (Bowers et al., 2005).

The carboxylate groups of the DNBA and DNSA anions in both (II) and (III) are also essentially coplanar with the benzene rings: torsion angles $\mathrm{C} 2 B-\mathrm{C} 1 B-\mathrm{C} 11 B-\mathrm{O} 11 B=$ -176.60 (16) and $-179.4(2)^{\circ}$, respectively. The 5- and 3substituted nitro groups are also either in-plane or out-ofplane [torsion angles $\mathrm{C} 4 B-\mathrm{C} 5 B-\mathrm{N} 5 B-\mathrm{O} 52 B=$ $179.61(16)^{\circ}$ in (II) and -177.5 (2) in (III) and $\mathrm{C} 2 B-\mathrm{C} 3 B-$ $\mathrm{N} 3 B-\mathrm{O} 32 B=-166.31(17)^{\circ}$ in (II) and $-155.2(2)^{\circ}$ in (III)]. Also, in (III), the phenolic substituent group (O2B) is disordered by rotation about the $\mathrm{C} 1 B \cdots \mathrm{C} 4 B$ ring vector giving a minor site-occupancy factor for the $\mathrm{O} 21 B-\mathrm{H} 21 B$ group of 0.28 (SOF fixed in the final refinement cycles). This is similar to the disorder in three examples among the DNSA protontransfer salts with Lewis bases, e.g. with nicotinamide ( $\mathrm{SOF}=$ 0.76/0.24) (Koman et al., 2003), with 2,6-diaminopyridine $(0.90 / 0.10)$ (Smith et al., 2003) and with quinoline-2-carboxylic acid (0.51/0.49) (Smith et al., 2007). In (III), the usual short intramolecular phenol $\mathrm{O}-\mathrm{H} \cdots \mathrm{O}_{\text {carboxyl }}$ hydrogen bond is present (Table 3).

\section{Supramolecular features}

In the crystal of (I), the $\mathrm{N} 8 A-\mathrm{H} \cdots \mathrm{O} 11 B$ hydrogen-bonded cation-anion pairs are extended through intermolecular $\mathrm{N} 4 B-\mathrm{H} \cdots \mathrm{O} 11 B^{\mathrm{i}}$ and $\cdots \mathrm{N} 12 B^{\mathrm{ii}}$ hydrogen-bonding extensions (Table 1), giving an overall three-dimensional network structure (Fig. 4). The structure contains no inter-ring $\pi-\pi$ interactions or $\mathrm{C}-\mathrm{H} \cdots \mathrm{O}$ hydrogen bonds.

The unit-cell parameters, space group (Table 4), and the overall crystal packing of (II) and (III) are very similar (Figs. 5 and 6). Although no classical hydrogen-bonding interactions are present between the primary cation-anion pairs, with both structures there are two minor cation $\mathrm{C}-\mathrm{H} \cdots \mathrm{O}$ hydrogenbonding extensions to nitro O-atom acceptors, $\mathrm{C} 2 \mathrm{~A}-$ $\mathrm{H} \cdots \mathrm{O} 31 B^{\mathrm{ii}}[3.309$ (2) $\AA$ in (II) and 3.281 (3) $\AA$ in (III)] and $\mathrm{C} 10 A-\mathrm{H} \cdots \mathrm{O} 32 B^{\mathrm{i}}[3.247$ (3) $\AA$ in (II) and 3.251 (5) $\AA$ in (III)] (Tables 2 and 3 ). These give two-dimensional layered structures lying parallel to (001). There are no inter-ring $\pi-\pi$ interactions in either (II) or (III).

\section{Synthesis and crystallization}

The title compounds (I)-(III) were prepared by first dissolving $100 \mathrm{mg}$ of either PABA, DNBA, or DNSA in $5 \mathrm{~mL}$ of warm 
ethanol followed by the addition, with stirring, of $111 \mathrm{mg}$ (I), $72 \mathrm{mg}$ (II) or $67 \mathrm{mg}$ (III) of BDU, respectively. Slow evaporation at room temperature gave colourless needles of (I), colourless prisms of (II), and fine yellow needles of (III), from which specimens were cleaved for the X-ray analyses.

\section{Refinement details}

Crystal data, data collection and structure refinement details are given in Table 4. Hydrogen atoms were placed in calculated positions $\left[\mathrm{C}-\mathrm{H}_{\text {aromatic }}=0.95 \AA\right.$ or $\mathrm{C}-\mathrm{H}_{\text {methylene }}=$ $0.99 \AA]$ and were allowed to ride in the refinements, with $U_{\text {iso }}(\mathrm{H})=1.2 U_{\text {eq }}(\mathrm{C})$. The amine and aminium $\mathrm{H}$-atoms were located in difference-Fourier analyses and were allowed to refine with distance restraints $[\mathrm{N}-\mathrm{H}=0.90$ (2) $\AA]$ and with $U_{\text {iso }}(\mathrm{H})=1.2 U_{\text {eq }}(\mathrm{N})$. Disorder involving atoms C9 $A$ and $\mathrm{C} 10 A$ of the six-membered ring systems of both (II) and (III) gave refined minor occupancy sites $\mathrm{C} 12 A$ and $\mathrm{C} 13 A$, with site occupancy factors of $0.735(3) / 0.265(3)$ and $0.686(4) /$ 0.314 (4), respectively. Also in (III), the phenol group of the DNSA anion was found to be disordered with the minor occupancy site $(\mathrm{O} 21 B)$ having a $\mathrm{SOF}=0.28$, which was fixed in the final cycles of refinement. In the structure of (I), although of no relevance in the achiral molecule, the Flack parameter (Flack, 1983) was determined as -0.1 (13) for 1668 Friedel pairs, which serves to indicate the lack of any usable anomalous scattering signal, as expected for an all-light-atom structure determined with Mo $K \alpha \mathrm{X}$-rays.

\section{Acknowledgements}

GS acknowledges financial support from the Science and Engineering Faculty, Queensland University of Technology.

\section{References}

Agilent (2014). CrysAlis PRO. Agilent Technologies Ltd, Yarnton, England.

Altomare, A., Cascarano, G., Giacovazzo, C. \& Guagliardi, A. (1993). J. Appl. Cryst. 26, 343-350.

Bowers, J. R., Hopkins, G. W., Yap, G. P. A. \& Wheeler, K. A. (2005). Cryst. Growth Des. 5, 727-736.

Cordiner, R. L., Hill, A. F. \& Wagler, J. (2008). Organometallics, 27, 4532-4540.

Farrugia, L. J. (2012). J. Appl. Cryst. 45, 849-854.

Flack, H. D. (1983). Acta Cryst. A39, 876-881.

Gracin, S. \& Fischer, A. (2005). Acta Cryst. E61, o1242-o1244.

Groom, C. R. \& Allen, F. H. (2014). Angew. Chem. Int. Ed. 53, 662671.

Heldebrant, D. J., Yonker, C. R., Jessop, P. G. \& Phan, L. (2009). Chem. Eur. J. 15, 7619-7627.

Huczyński, A., Ratajczak-Sitarz, M., Katrusiak, A. \& Brzezinski, B. (2008). J. Mol. Struct. 889, 64-71.

Koman, M., Martiška, L., Valigura, D. \& Glowiak, T. (2003). Acta Cryst. E59, o441-0442.

Lipstman, S. \& Goldberg, I. (2013). Cryst. Growth Des. 13, 942952.

Lynch, D. E. \& McClenaghan, I. (2003). Cryst. Eng. 6, 99-107.

Motevalli, M., Hursthouse, M. B., Kelly, P. F. \& Woollins, J. D. (1989). Polyhedron, 8, 893-896.

Pérez, E. R., Santos, R. H. A., Gambardella, M. T. P., de Macedo, L. G. M., Rodrigues-Filho, U. P., Launay, J. \& Franco, D. W. (2004). J. Org. Chem. 69, 8005-8011.

Regalado, E. L., Mendiola, J., Laguna, A., Nogueiras, C. \& Thomas, O. P. (2010). Nat. Prod. Commun. 5, 1187-1190.

Saftić, D., Žinić, B. \& Višnjevac, A. (2012). Tetrahedron, 68, 10621070.

Shannon, M. S., Irvin, A. C., Liu, H., Moon, J. D., Hindman, M. S., Turner, C. H. \& Bara, J. E. (2015). Ind. Eng. Chem. Res. 54, 462471.

Sheldrick, G. M. (2008). Acta Cryst. A64, 112-122.

Smith, G., Wermuth, U. D., Healy, P. C. \& White, J. M. (2003). Aust. J. Chem. 56, 707-713.

Smith, G., Wermuth, U. D., Healy, P. C. \& White, J. M. (2007). Aust. J. Chem. 60, 264-277.

Spek, A. L. (2009). Acta Cryst. D65, 148-155. 


\section{supporting information}

Acta Cryst. (2016). E72, 382-386 [doi:10.1107/S205698901600267X]

Crystal structures of three anhydrous salts of the Lewis base 1,8-diazabicyclo[5.4.0] undec-7-ene (DBU) with the ring-substituted benzoic acid analogues 4aminobenzoic acid, 3,5-dinitrobenzoic acid and 3,5-dinitrosalicylic acid

\section{Graham Smith and Daniel E. Lynch}

\section{Computing details}

For all compounds, data collection: CrysAlis PRO (Agilent, 2014); cell refinement: CrysAlis PRO (Agilent, 2014); data reduction: CrysAlis PRO (Agilent, 2014); program(s) used to solve structure: SIR92 (Altomare et al., 1993); program(s) used to refine structure: SHELXL97 (Sheldrick, 2008) within WinGX (Farrugia, 2012); molecular graphics: PLATON (Spek, 2009); software used to prepare material for publication: PLATON (Spek, 2009).

(I) 1-Aza-8-azoniabicyclo[5.4.0] undec-7-ene 4-aminobenzoate

Crystal data

$\mathrm{C}_{9} \mathrm{H}_{17} \mathrm{~N}_{2}^{+} \cdot \mathrm{C}_{7} \mathrm{H}_{6} \mathrm{NO}_{2}^{-}$

$M_{r}=289.37$

Orthorhombic, $P 2_{1} 2_{1} 2_{1}$

Hall symbol: P 2ac 2ab

$a=8.0986(4) \AA$

$b=12.9213(6) \AA$

$c=13.7344(7) \AA$

$V=1437.23(12) \AA^{3}$

$Z=4$

Data collection

Oxford Diffraction Gemini-S CCD-detector diffractometer

Radiation source: Enhance (Mo) X-ray source

Graphite monochromator

Detector resolution: 16.067 pixels $\mathrm{mm}^{-1}$

$\omega$ scans

Absorption correction: multi-scan

(CrysAlis PRO; Agilent, 2014)

$T_{\min }=0.93, T_{\max }=0.99$

\section{Refinement}

Refinement on $F^{2}$

Least-squares matrix: full

$R\left[F^{2}>2 \sigma\left(F^{2}\right)\right]=0.044$

$w R\left(F^{2}\right)=0.098$

$S=1.07$

3324 reflections

199 parameters
$F(000)=624$

$D_{\mathrm{x}}=1.337 \mathrm{Mg} \mathrm{m}^{-3}$

Mo $K \alpha$ radiation, $\lambda=0.71073 \AA$

Cell parameters from 2097 reflections

$\theta=3.5-28.4^{\circ}$

$\mu=0.09 \mathrm{~mm}^{-1}$

$T=200 \mathrm{~K}$

Prism, colourless

$0.40 \times 0.26 \times 0.24 \mathrm{~mm}$

7372 measured reflections

3324 independent reflections

2847 reflections with $I>2 \sigma(I)$

$R_{\text {int }}=0.031$

$\theta_{\max }=29.2^{\circ}, \theta_{\min }=3.3^{\circ}$

$h=-10 \rightarrow 10$

$k=-16 \rightarrow 15$

$l=-17 \rightarrow 18$

3 restraints

Primary atom site location: structure-invariant direct methods

Secondary atom site location: difference Fourier map

Hydrogen site location: inferred from neighbouring sites 
$\mathrm{H}$ atoms treated by a mixture of independent and constrained refinement

$w=1 /\left[\sigma^{2}\left(F_{\mathrm{o}}^{2}\right)+(0.0438 P)^{2}+0.0476 P\right]$

where $P=\left(F_{\mathrm{o}}^{2}+2 F_{\mathrm{c}}^{2}\right) / 3$

$(\Delta / \sigma)_{\max }<0.001$
$\Delta \rho_{\max }=0.20 \mathrm{e} \AA^{-3}$

$\Delta \rho_{\min }=-0.25$ e $\AA^{-3}$

Absolute structure: Flack (1983), 1668 Friedel pairs

Absolute structure parameter: -0.1 (13)

Special details

Geometry. Bond distances, angles etc. have been calculated using the rounded fractional coordinates. All su's are estimated from the variances of the (full) variance-covariance matrix. The cell esds are taken into account in the estimation of distances, angles and torsion angles

Refinement. Refinement of $\mathrm{F}^{2}$ against ALL reflections. The weighted R-factor $\mathrm{wR}$ and goodness of fit $\mathrm{S}$ are based on $\mathrm{F}^{2}$, conventional R-factors $R$ are based on $F$, with $F$ set to zero for negative $F^{2}$. The threshold expression of $F^{2}>2 \operatorname{sigma}\left(\mathrm{F}^{2}\right)$ is used only for calculating R-factors(gt) etc. and is not relevant to the choice of reflections for refinement. R-factors based on $\mathrm{F}^{2}$ are statistically about twice as large as those based on F, and R- factors based on ALL data will be even larger.

Fractional atomic coordinates and isotropic or equivalent isotropic displacement parameters $\left(\AA^{2}\right)$

\begin{tabular}{|c|c|c|c|c|}
\hline & $x$ & $y$ & $z$ & $U_{\text {iso }} * / U_{\text {eq }}$ \\
\hline N1A & $0.32105(18)$ & $0.84571(11)$ & $0.67893(11)$ & $0.0229(4)$ \\
\hline N8A & $0.36282(18)$ & $0.67732(12)$ & $0.62864(11)$ & $0.0241(5)$ \\
\hline $\mathrm{C} 2 \mathrm{~A}$ & $0.2390(2)$ & $0.94651(14)$ & $0.66676(13)$ & $0.0256(5)$ \\
\hline $\mathrm{C} 3 \mathrm{~A}$ & $0.3174(2)$ & $1.01454(14)$ & $0.58999(14)$ & $0.0291(6)$ \\
\hline $\mathrm{C} 4 \mathrm{~A}$ & $0.2728(2)$ & $0.98456(14)$ & $0.48576(14)$ & $0.0288(6)$ \\
\hline $\mathrm{C} 5 \mathrm{~A}$ & 0.3145 & $0.87339(14)$ & $0.45932(13)$ & $0.0271(5)$ \\
\hline C6A & $0.2207(2)$ & $0.79201(14)$ & $0.51882(13)$ & $0.0262(5)$ \\
\hline $\mathrm{C} 7 \mathrm{~A}$ & $0.3028(2)$ & $0.77086(13)$ & $0.61456(13)$ & $0.0209(5)$ \\
\hline $\mathrm{C} 9 \mathrm{~A}$ & $0.4591(2)$ & $0.64922(14)$ & $0.71447(13)$ & $0.0262(5)$ \\
\hline C10A & $0.5429(2)$ & $0.74497(13)$ & $0.75333(13)$ & $0.0280(6)$ \\
\hline C11A & $0.4170(2)$ & $0.82988(15)$ & $0.76868(13)$ & $0.0302(6)$ \\
\hline O11B & $0.28719(17)$ & $0.51621(9)$ & $0.51597(9)$ & $0.0320(4)$ \\
\hline O12B & 0.29529 (19) & $0.56473(11)$ & $0.36120(11)$ & $0.0428(5)$ \\
\hline N4B & $0.6170(2)$ & $0.11141(13)$ & $0.33808(12)$ & $0.0296(5)$ \\
\hline $\mathrm{C} 1 \mathrm{~B}$ & $0.3958(2)$ & $0.39741(13)$ & $0.40190(12)$ & $0.0206(5)$ \\
\hline $\mathrm{C} 2 \mathrm{~B}$ & $0.43611(19)$ & $0.36990(13)$ & $0.30648(12)$ & $0.0212(5)$ \\
\hline $\mathrm{C} 3 \mathrm{~B}$ & $0.5089(2)$ & $0.27615(13)$ & $0.28504(12)$ & $0.0220(5)$ \\
\hline $\mathrm{C} 4 \mathrm{~B}$ & $0.5475(2)$ & $0.20495(13)$ & $0.35867(13)$ & $0.0220(5)$ \\
\hline $\mathrm{C} 5 \mathrm{~B}$ & $0.5100(2)$ & $0.23325(13)$ & $0.45489(12)$ & $0.0243(5)$ \\
\hline C6B & $0.4347(2)$ & $0.32664(13)$ & $0.47496(13)$ & $0.0227(5)$ \\
\hline C11B & $0.3204(2)$ & $0.50006(14)$ & $0.42672(14)$ & $0.0238(5)$ \\
\hline H8A & $0.342(2)$ & $0.6279(14)$ & $0.5850(13)$ & $0.0290^{*}$ \\
\hline H10A & 0.59810 & 0.72880 & 0.81580 & $0.0340^{*}$ \\
\hline H11A & 0.34180 & 0.81070 & 0.82260 & $0.0360^{*}$ \\
\hline H12A & 0.47390 & 0.89490 & 0.78670 & $0.0360 *$ \\
\hline H13A & 0.62810 & 0.76860 & 0.70660 & $0.0340 *$ \\
\hline $\mathrm{H} 21 \mathrm{~A}$ & 0.24080 & 0.98360 & 0.72980 & $0.0310^{*}$ \\
\hline $\mathrm{H} 22 \mathrm{~A}$ & 0.12200 & 0.93460 & 0.64920 & $0.0310^{*}$ \\
\hline H31A & 0.43890 & 1.01140 & 0.59740 & $0.0350^{*}$ \\
\hline H32A & 0.28290 & 1.08700 & 0.60140 & $0.0350^{*}$ \\
\hline H41A & 0.15290 & 0.99540 & 0.47620 & $0.0350 *$ \\
\hline
\end{tabular}




\begin{tabular}{lllll} 
H42A & 0.33170 & 1.03140 & 0.44050 & $0.0350^{*}$ \\
H51A & 0.43450 & 0.86260 & 0.46870 & $0.0320^{*}$ \\
H52A & 0.29000 & 0.86260 & 0.38940 & $0.0320^{*}$ \\
H61A & 0.10660 & 0.81660 & 0.53060 & $0.0310^{*}$ \\
H62A & 0.21410 & 0.72690 & 0.48100 & $0.0310^{*}$ \\
H91A & 0.54280 & 0.59660 & 0.69700 & $0.0310^{*}$ \\
H92A & 0.38570 & 0.61950 & 0.76490 & $0.0310^{*}$ \\
H2B & 0.41290 & 0.41690 & 0.25510 & $0.0250^{*}$ \\
H3B & 0.53320 & 0.25950 & 0.21920 & $0.0260^{*}$ \\
H5B & 0.53680 & 0.18760 & 0.50670 & $0.0290^{*}$ \\
H6B & 0.40870 & 0.34320 & 0.54060 & $0.0270^{*}$ \\
H41B & $0.666(2)$ & $0.0742(16)$ & $0.3845(13)$ & $0.0360^{*}$ \\
H42B & $0.647(2)$ & $0.0939(16)$ & $0.2759(12)$ & $0.0360^{*}$ \\
\hline
\end{tabular}

Atomic displacement parameters $\left(\AA^{2}\right)$

\begin{tabular}{lllllll}
\hline & $U^{11}$ & $U^{22}$ & $U^{33}$ & $U^{12}$ & $U^{13}$ & $U^{23}$ \\
\hline N1A & $0.0263(7)$ & $0.0220(8)$ & $0.0205(7)$ & $0.0030(6)$ & $-0.0033(6)$ & $-0.0022(6)$ \\
N8A & $0.0294(8)$ & $0.0197(8)$ & $0.0233(8)$ & $-0.0004(6)$ & $-0.0008(6)$ & $-0.0024(7)$ \\
C2A & $0.0276(9)$ & $0.0223(9)$ & $0.0269(10)$ & $0.0053(7)$ & $-0.0018(7)$ & $-0.0053(8)$ \\
C3A & $0.0311(10)$ & $0.0218(9)$ & $0.0343(11)$ & $-0.0005(8)$ & $-0.0031(8)$ & $-0.0024(8)$ \\
C4A & $0.0314(10)$ & $0.0263(9)$ & $0.0287(10)$ & $0.0011(8)$ & $-0.0008(8)$ & $0.0041(8)$ \\
C5A & $0.0295(9)$ & $0.0292(10)$ & $0.0225(9)$ & $0.0039(8)$ & $-0.0010(7)$ & $-0.0007(8)$ \\
C6A & $0.0312(9)$ & $0.0221(9)$ & $0.0253(9)$ & $-0.0010(7)$ & $-0.0062(8)$ & $-0.0035(8)$ \\
C7A & $0.0202(8)$ & $0.0203(9)$ & $0.0223(9)$ & $-0.0013(7)$ & $0.0013(7)$ & $-0.0012(7)$ \\
C9A & $0.0268(9)$ & $0.0251(9)$ & $0.0267(10)$ & $0.0030(8)$ & $-0.0003(7)$ & $0.0033(8)$ \\
C10A & $0.0269(9)$ & $0.0301(10)$ & $0.0269(10)$ & $0.0034(8)$ & $-0.0065(8)$ & $-0.0010(8)$ \\
C11A & $0.0365(11)$ & $0.0306(10)$ & $0.0235(9)$ & $0.0053(8)$ & $-0.0094(8)$ & $-0.0056(8)$ \\
O11B & $0.0520(8)$ & $0.0207(7)$ & $0.0233(7)$ & $-0.0025(6)$ & $0.0074(6)$ & $-0.0035(5)$ \\
O12B & $0.0643(9)$ & $0.0337(8)$ & $0.0305(8)$ & $0.0187(7)$ & $0.0123(7)$ & $0.0099(7)$ \\
N4B & $0.0406(9)$ & $0.0260(9)$ & $0.0223(9)$ & $0.0072(7)$ & $0.0000(7)$ & $-0.0008(7)$ \\
C1B & $0.0194(8)$ & $0.0215(9)$ & $0.0209(9)$ & $-0.0045(6)$ & $-0.0004(7)$ & $0.0005(7)$ \\
C2B & $0.0225(9)$ & $0.0237(9)$ & $0.0174(8)$ & $-0.0015(7)$ & $-0.0013(6)$ & $0.0033(7)$ \\
C3B & $0.0240(8)$ & $0.0241(8)$ & $0.0179(8)$ & $-0.0032(7)$ & $0.0001(7)$ & $0.0010(7)$ \\
C4B & $0.0216(8)$ & $0.0195(9)$ & $0.0250(9)$ & $-0.0025(7)$ & $-0.0011(7)$ & $-0.0019(7)$ \\
C5B & $0.0322(9)$ & $0.0221(9)$ & $0.0185(8)$ & $0.0004(8)$ & $-0.0011(7)$ & $0.0046(7)$ \\
C6B & $0.0288(9)$ & $0.0228(9)$ & $0.0166(8)$ & $-0.0043(7)$ & $0.0018(7)$ & $-0.0017(7)$ \\
C11B & $0.0255(9)$ & $0.0221(9)$ & $0.0237(9)$ & $-0.0043(7)$ & $0.0028(7)$ & $0.0001(7)$ \\
& & & & & &
\end{tabular}

Geometric parameters $(\AA, \stackrel{\circ}{)})$

\begin{tabular}{|c|c|c|c|}
\hline $\mathrm{O} 11 \mathrm{~B}-\mathrm{C} 11 \mathrm{~B}$ & $1.272(2)$ & $\mathrm{C} 4 \mathrm{~A}-\mathrm{H} 41 \mathrm{~A}$ & 0.9900 \\
\hline $\mathrm{O} 12 \mathrm{~B}-\mathrm{C} 11 \mathrm{~B}$ & $1.245(2)$ & $\mathrm{C} 5 \mathrm{~A}-\mathrm{H} 52 \mathrm{~A}$ & 0.9900 \\
\hline $\mathrm{N} 1 \mathrm{~A}-\mathrm{C} 11 \mathrm{~A}$ & $1.471(2)$ & $\mathrm{C} 5 \mathrm{~A}-\mathrm{H} 51 \mathrm{~A}$ & 0.9900 \\
\hline $\mathrm{N} 1 \mathrm{~A}-\mathrm{C} 7 \mathrm{~A}$ & $1.319(2)$ & C6A-H61A & 0.9900 \\
\hline $\mathrm{N} 1 \mathrm{~A}-\mathrm{C} 2 \mathrm{~A}$ & $1.472(2)$ & $\mathrm{C} 6 \mathrm{~A}-\mathrm{H} 62 \mathrm{~A}$ & 0.9900 \\
\hline $\mathrm{N} 8 \mathrm{~A}-\mathrm{C} 7 \mathrm{~A}$ & $1.317(2)$ & C9A-H91A & 0.9900 \\
\hline $\mathrm{N} 8 \mathrm{~A}-\mathrm{C} 9 \mathrm{~A}$ & $1.459(2)$ & $\mathrm{C} 9 \mathrm{~A}-\mathrm{H} 92 \mathrm{~A}$ & 0.9900 \\
\hline
\end{tabular}




\begin{tabular}{|c|c|c|c|}
\hline $\mathrm{N} 8 \mathrm{~A}-\mathrm{H} 8 \mathrm{~A}$ & $0.892(18)$ & $\mathrm{C} 10 \mathrm{~A}-\mathrm{H} 10 \mathrm{~A}$ & 0.9900 \\
\hline $\mathrm{N} 4 \mathrm{~B}-\mathrm{C} 4 \mathrm{~B}$ & $1.363(2)$ & $\mathrm{C} 10 \mathrm{~A}-\mathrm{H} 13 \mathrm{~A}$ & 0.9900 \\
\hline $\mathrm{N} 4 \mathrm{~B}-\mathrm{H} 41 \mathrm{~B}$ & $0.892(18)$ & $\mathrm{C} 11 \mathrm{~A}-\mathrm{H} 12 \mathrm{~A}$ & 0.9900 \\
\hline $\mathrm{N} 4 \mathrm{~B}-\mathrm{H} 42 \mathrm{~B}$ & $0.916(17)$ & $\mathrm{C} 11 \mathrm{~A}-\mathrm{H} 11 \mathrm{~A}$ & 0.9900 \\
\hline $\mathrm{C} 2 \mathrm{~A}-\mathrm{C} 3 \mathrm{~A}$ & $1.513(3)$ & $\mathrm{C} 1 \mathrm{~B}-\mathrm{C} 11 \mathrm{~B}$ & $1.499(2)$ \\
\hline $\mathrm{C} 3 \mathrm{~A}-\mathrm{C} 4 \mathrm{~A}$ & $1.526(3)$ & $\mathrm{C} 1 \mathrm{~B}-\mathrm{C} 2 \mathrm{~B}$ & $1.397(2)$ \\
\hline $\mathrm{C} 4 \mathrm{~A}-\mathrm{C} 5 \mathrm{~A}$ & $1.520(3)$ & $\mathrm{C} 1 \mathrm{~B}-\mathrm{C} 6 \mathrm{~B}$ & $1.394(2)$ \\
\hline $\mathrm{C} 5 \mathrm{~A}-\mathrm{C} 6 \mathrm{~A}$ & $1.533(2)$ & $\mathrm{C} 2 \mathrm{~B}-\mathrm{C} 3 \mathrm{~B}$ & $1.379(2)$ \\
\hline $\mathrm{C} 6 \mathrm{~A}-\mathrm{C} 7 \mathrm{~A}$ & $1.499(2)$ & $\mathrm{C} 3 \mathrm{~B}-\mathrm{C} 4 \mathrm{~B}$ & $1.402(2)$ \\
\hline $\mathrm{C} 9 \mathrm{~A}-\mathrm{C} 10 \mathrm{~A}$ & $1.509(2)$ & $\mathrm{C} 4 \mathrm{~B}-\mathrm{C} 5 \mathrm{~B}$ & $1.404(2)$ \\
\hline $\mathrm{C} 10 \mathrm{~A}-\mathrm{C} 11 \mathrm{~A}$ & $1.513(2)$ & $\mathrm{C} 5 \mathrm{~B}-\mathrm{C} 6 \mathrm{~B}$ & $1.380(2)$ \\
\hline $\mathrm{C} 2 \mathrm{~A}-\mathrm{H} 21 \mathrm{~A}$ & 0.9900 & $\mathrm{C} 2 \mathrm{~B}-\mathrm{H} 2 \mathrm{~B}$ & 0.9500 \\
\hline $\mathrm{C} 2 \mathrm{~A}-\mathrm{H} 22 \mathrm{~A}$ & 0.9900 & $\mathrm{C} 3 \mathrm{~B}-\mathrm{H} 3 \mathrm{~B}$ & 0.9500 \\
\hline $\mathrm{C} 3 \mathrm{~A}-\mathrm{H} 31 \mathrm{~A}$ & 0.9900 & $\mathrm{C} 5 \mathrm{~B}-\mathrm{H} 5 \mathrm{~B}$ & 0.9500 \\
\hline $\mathrm{C} 3 \mathrm{~A}-\mathrm{H} 32 \mathrm{~A}$ & 0.9900 & $\mathrm{C} 6 \mathrm{~B}-\mathrm{H} 6 \mathrm{~B}$ & 0.9500 \\
\hline $\mathrm{C} 4 \mathrm{~A}-\mathrm{H} 42 \mathrm{~A}$ & 0.9900 & & \\
\hline $\mathrm{C} 2 \mathrm{~A}-\mathrm{N} 1 \mathrm{~A}-\mathrm{C} 7 \mathrm{~A}$ & $121.49(15)$ & $\mathrm{C} 5 \mathrm{~A}-\mathrm{C} 6 \mathrm{~A}-\mathrm{H} 62 \mathrm{~A}$ & 109.00 \\
\hline $\mathrm{C} 2 \mathrm{~A}-\mathrm{N} 1 \mathrm{~A}-\mathrm{C} 11 \mathrm{~A}$ & $117.17(14)$ & $\mathrm{H} 61 \mathrm{~A}-\mathrm{C} 6 \mathrm{~A}-\mathrm{H} 62 \mathrm{~A}$ & 108.00 \\
\hline $\mathrm{C} 7 \mathrm{~A}-\mathrm{N} 1 \mathrm{~A}-\mathrm{C} 11 \mathrm{~A}$ & $121.26(15)$ & $\mathrm{C} 7 \mathrm{~A}-\mathrm{C} 6 \mathrm{~A}-\mathrm{H} 62 \mathrm{~A}$ & 109.00 \\
\hline $\mathrm{C} 7 \mathrm{~A}-\mathrm{N} 8 \mathrm{~A}-\mathrm{C} 9 \mathrm{~A}$ & $122.97(15)$ & $\mathrm{C} 7 \mathrm{~A}-\mathrm{C} 6 \mathrm{~A}-\mathrm{H} 61 \mathrm{~A}$ & 109.00 \\
\hline $\mathrm{C} 7 \mathrm{~A}-\mathrm{N} 8 \mathrm{~A}-\mathrm{H} 8 \mathrm{~A}$ & $119.3(11)$ & $\mathrm{C} 10 \mathrm{~A}-\mathrm{C} 9 \mathrm{~A}-\mathrm{H} 91 \mathrm{~A}$ & 110.00 \\
\hline $\mathrm{C} 9 \mathrm{~A}-\mathrm{N} 8 \mathrm{~A}-\mathrm{H} 8 \mathrm{~A}$ & $117.8(12)$ & $\mathrm{N} 8 \mathrm{~A}-\mathrm{C} 9 \mathrm{~A}-\mathrm{H} 92 \mathrm{~A}$ & 110.00 \\
\hline $\mathrm{C} 4 \mathrm{~B}-\mathrm{N} 4 \mathrm{~B}-\mathrm{H} 41 \mathrm{~B}$ & $120.9(13)$ & $\mathrm{N} 8 \mathrm{~A}-\mathrm{C} 9 \mathrm{~A}-\mathrm{H} 91 \mathrm{~A}$ & 110.00 \\
\hline $\mathrm{H} 41 \mathrm{~B}-\mathrm{N} 4 \mathrm{~B}-\mathrm{H} 42 \mathrm{~B}$ & $114.5(17)$ & $\mathrm{C} 10 \mathrm{~A}-\mathrm{C} 9 \mathrm{~A}-\mathrm{H} 92 \mathrm{~A}$ & 110.00 \\
\hline $\mathrm{C} 4 \mathrm{~B}-\mathrm{N} 4 \mathrm{~B}-\mathrm{H} 42 \mathrm{~B}$ & $121.5(13)$ & $\mathrm{H} 91 \mathrm{~A}-\mathrm{C} 9 \mathrm{~A}-\mathrm{H} 92 \mathrm{~A}$ & 108.00 \\
\hline $\mathrm{N} 1 \mathrm{~A}-\mathrm{C} 2 \mathrm{~A}-\mathrm{C} 3 \mathrm{~A}$ & $113.83(14)$ & $\mathrm{C} 9 \mathrm{~A}-\mathrm{C} 10 \mathrm{~A}-\mathrm{H} 10 \mathrm{~A}$ & 110.00 \\
\hline $\mathrm{C} 2 \mathrm{~A}-\mathrm{C} 3 \mathrm{~A}-\mathrm{C} 4 \mathrm{~A}$ & $114.02(15)$ & $\mathrm{H} 10 \mathrm{~A}-\mathrm{C} 10 \mathrm{~A}-\mathrm{H} 13 \mathrm{~A}$ & 108.00 \\
\hline $\mathrm{C} 3 \mathrm{~A}-\mathrm{C} 4 \mathrm{~A}-\mathrm{C} 5 \mathrm{~A}$ & $114.29(15)$ & $\mathrm{C} 9 \mathrm{~A}-\mathrm{C} 10 \mathrm{~A}-\mathrm{H} 13 \mathrm{~A}$ & 110.00 \\
\hline $\mathrm{C} 4 \mathrm{~A}-\mathrm{C} 5 \mathrm{~A}-\mathrm{C} 6 \mathrm{~A}$ & $114.26(14)$ & $\mathrm{C} 11 \mathrm{~A}-\mathrm{C} 10 \mathrm{~A}-\mathrm{H} 10 \mathrm{~A}$ & 110.00 \\
\hline $\mathrm{C} 5 \mathrm{~A}-\mathrm{C} 6 \mathrm{~A}-\mathrm{C} 7 \mathrm{~A}$ & $111.90(14)$ & $\mathrm{C} 11 \mathrm{~A}-\mathrm{C} 10 \mathrm{~A}-\mathrm{H} 13 \mathrm{~A}$ & 110.00 \\
\hline $\mathrm{N} 8 \mathrm{~A}-\mathrm{C} 7 \mathrm{~A}-\mathrm{C} 6 \mathrm{~A}$ & $117.38(15)$ & $\mathrm{N} 1 \mathrm{~A}-\mathrm{C} 11 \mathrm{~A}-\mathrm{H} 11 \mathrm{~A}$ & 110.00 \\
\hline $\mathrm{N} 1 \mathrm{~A}-\mathrm{C} 7 \mathrm{~A}-\mathrm{N} 8 \mathrm{~A}$ & $122.23(16)$ & $\mathrm{N} 1 \mathrm{~A}-\mathrm{C} 11 \mathrm{~A}-\mathrm{H} 12 \mathrm{~A}$ & 110.00 \\
\hline $\mathrm{N} 1 \mathrm{~A}-\mathrm{C} 7 \mathrm{~A}-\mathrm{C} 6 \mathrm{~A}$ & $120.28(15)$ & $\mathrm{C} 10 \mathrm{~A}-\mathrm{C} 11 \mathrm{~A}-\mathrm{H} 11 \mathrm{~A}$ & 110.00 \\
\hline $\mathrm{N} 8 \mathrm{~A}-\mathrm{C} 9 \mathrm{~A}-\mathrm{C} 10 \mathrm{~A}$ & $108.79(14)$ & $\mathrm{H} 11 \mathrm{~A}-\mathrm{C} 11 \mathrm{~A}-\mathrm{H} 12 \mathrm{~A}$ & 108.00 \\
\hline $\mathrm{C} 9 \mathrm{~A}-\mathrm{C} 10 \mathrm{~A}-\mathrm{C} 11 \mathrm{~A}$ & $109.93(14)$ & $\mathrm{C} 10 \mathrm{~A}-\mathrm{C} 11 \mathrm{~A}-\mathrm{H} 12 \mathrm{~A}$ & 110.00 \\
\hline $\mathrm{N} 1 \mathrm{~A}-\mathrm{C} 11 \mathrm{~A}-\mathrm{C} 10 \mathrm{~A}$ & $109.88(14)$ & $\mathrm{C} 6 \mathrm{~B}-\mathrm{C} 1 \mathrm{~B}-\mathrm{C} 11 \mathrm{~B}$ & $120.58(15)$ \\
\hline $\mathrm{C} 3 \mathrm{~A}-\mathrm{C} 2 \mathrm{~A}-\mathrm{H} 22 \mathrm{~A}$ & 109.00 & $\mathrm{C} 2 \mathrm{~B}-\mathrm{C} 1 \mathrm{~B}-\mathrm{C} 11 \mathrm{~B}$ & $122.25(15)$ \\
\hline $\mathrm{H} 21 \mathrm{~A}-\mathrm{C} 2 \mathrm{~A}-\mathrm{H} 22 \mathrm{~A}$ & 108.00 & $\mathrm{C} 2 \mathrm{~B}-\mathrm{C} 1 \mathrm{~B}-\mathrm{C} 6 \mathrm{~B}$ & $117.12(15)$ \\
\hline $\mathrm{N} 1 \mathrm{~A}-\mathrm{C} 2 \mathrm{~A}-\mathrm{H} 22 \mathrm{~A}$ & 109.00 & $\mathrm{C} 1 \mathrm{~B}-\mathrm{C} 2 \mathrm{~B}-\mathrm{C} 3 \mathrm{~B}$ & $121.59(15)$ \\
\hline $\mathrm{C} 3 \mathrm{~A}-\mathrm{C} 2 \mathrm{~A}-\mathrm{H} 21 \mathrm{~A}$ & 109.00 & $\mathrm{C} 2 \mathrm{~B}-\mathrm{C} 3 \mathrm{~B}-\mathrm{C} 4 \mathrm{~B}$ & $121.17(15)$ \\
\hline $\mathrm{N} 1 \mathrm{~A}-\mathrm{C} 2 \mathrm{~A}-\mathrm{H} 21 \mathrm{~A}$ & 109.00 & $\mathrm{~N} 4 \mathrm{~B}-\mathrm{C} 4 \mathrm{~B}-\mathrm{C} 3 \mathrm{~B}$ & $121.61(16)$ \\
\hline $\mathrm{C} 2 \mathrm{~A}-\mathrm{C} 3 \mathrm{~A}-\mathrm{H} 31 \mathrm{~A}$ & 109.00 & $\mathrm{~N} 4 \mathrm{~B}-\mathrm{C} 4 \mathrm{~B}-\mathrm{C} 5 \mathrm{~B}$ & $121.03(16)$ \\
\hline $\mathrm{C} 2 \mathrm{~A}-\mathrm{C} 3 \mathrm{~A}-\mathrm{H} 32 \mathrm{~A}$ & 109.00 & $\mathrm{C} 3 \mathrm{~B}-\mathrm{C} 4 \mathrm{~B}-\mathrm{C} 5 \mathrm{~B}$ & $117.36(15)$ \\
\hline $\mathrm{H} 31 \mathrm{~A}-\mathrm{C} 3 \mathrm{~A}-\mathrm{H} 32 \mathrm{~A}$ & 108.00 & $\mathrm{C} 4 \mathrm{~B}-\mathrm{C} 5 \mathrm{~B}-\mathrm{C} 6 \mathrm{~B}$ & $120.75(16)$ \\
\hline $\mathrm{C} 4 \mathrm{~A}-\mathrm{C} 3 \mathrm{~A}-\mathrm{H} 31 \mathrm{~A}$ & 109.00 & $\mathrm{C} 1 \mathrm{~B}-\mathrm{C} 6 \mathrm{~B}-\mathrm{C} 5 \mathrm{~B}$ & $122.00(16)$ \\
\hline $\mathrm{C} 4 \mathrm{~A}-\mathrm{C} 3 \mathrm{~A}-\mathrm{H} 32 \mathrm{~A}$ & 109.00 & $\mathrm{O} 11 \mathrm{~B}-\mathrm{C} 11 \mathrm{~B}-\mathrm{O} 12 \mathrm{~B}$ & $123.50(17)$ \\
\hline $\mathrm{C} 3 \mathrm{~A}-\mathrm{C} 4 \mathrm{~A}-\mathrm{H} 42 \mathrm{~A}$ & 109.00 & $\mathrm{O} 11 \mathrm{~B}-\mathrm{C} 11 \mathrm{~B}-\mathrm{C} 1 \mathrm{~B}$ & $116.76(16)$ \\
\hline
\end{tabular}




\begin{tabular}{|c|c|c|c|}
\hline $\mathrm{H} 41 \mathrm{~A}-\mathrm{C} 4 \mathrm{~A}-\mathrm{H} 42 \mathrm{~A}$ & 108.00 & $\mathrm{O} 12 \mathrm{~B}-\mathrm{C} 11 \mathrm{~B}-\mathrm{C} 1 \mathrm{~B}$ & $119.74(17)$ \\
\hline $\mathrm{C} 5 \mathrm{~A}-\mathrm{C} 4 \mathrm{~A}-\mathrm{H} 41 \mathrm{~A}$ & 109.00 & $\mathrm{C} 1 \mathrm{~B}-\mathrm{C} 2 \mathrm{~B}-\mathrm{H} 2 \mathrm{~B}$ & 119.00 \\
\hline $\mathrm{C} 5 \mathrm{~A}-\mathrm{C} 4 \mathrm{~A}-\mathrm{H} 42 \mathrm{~A}$ & 109.00 & $\mathrm{C} 3 \mathrm{~B}-\mathrm{C} 2 \mathrm{~B}-\mathrm{H} 2 \mathrm{~B}$ & 119.00 \\
\hline $\mathrm{C} 3 \mathrm{~A}-\mathrm{C} 4 \mathrm{~A}-\mathrm{H} 41 \mathrm{~A}$ & 109.00 & $\mathrm{C} 2 \mathrm{~B}-\mathrm{C} 3 \mathrm{~B}-\mathrm{H} 3 \mathrm{~B}$ & 119.00 \\
\hline $\mathrm{C} 6 \mathrm{~A}-\mathrm{C} 5 \mathrm{~A}-\mathrm{H} 52 \mathrm{~A}$ & 109.00 & $\mathrm{C} 4 \mathrm{~B}-\mathrm{C} 3 \mathrm{~B}-\mathrm{H} 3 \mathrm{~B}$ & 119.00 \\
\hline $\mathrm{H} 51 \mathrm{~A}-\mathrm{C} 5 \mathrm{~A}-\mathrm{H} 52 \mathrm{~A}$ & 108.00 & $\mathrm{C} 4 \mathrm{~B}-\mathrm{C} 5 \mathrm{~B}-\mathrm{H} 5 \mathrm{~B}$ & 120.00 \\
\hline $\mathrm{C} 4 \mathrm{~A}-\mathrm{C} 5 \mathrm{~A}-\mathrm{H} 52 \mathrm{~A}$ & 109.00 & $\mathrm{C} 6 \mathrm{~B}-\mathrm{C} 5 \mathrm{~B}-\mathrm{H} 5 \mathrm{~B}$ & 120.00 \\
\hline $\mathrm{C} 6 \mathrm{~A}-\mathrm{C} 5 \mathrm{~A}-\mathrm{H} 51 \mathrm{~A}$ & 109.00 & $\mathrm{C} 1 \mathrm{~B}-\mathrm{C} 6 \mathrm{~B}-\mathrm{H} 6 \mathrm{~B}$ & 119.00 \\
\hline $\mathrm{C} 4 \mathrm{~A}-\mathrm{C} 5 \mathrm{~A}-\mathrm{H} 51 \mathrm{~A}$ & 109.00 & $\mathrm{C} 5 \mathrm{~B}-\mathrm{C} 6 \mathrm{~B}-\mathrm{H} 6 \mathrm{~B}$ & 119.00 \\
\hline $\mathrm{C} 5 \mathrm{~A}-\mathrm{C} 6 \mathrm{~A}-\mathrm{H} 61 \mathrm{~A}$ & 109.00 & & \\
\hline $\mathrm{C} 7 \mathrm{~A}-\mathrm{N} 1 \mathrm{~A}-\mathrm{C} 2 \mathrm{~A}-\mathrm{C} 3 \mathrm{~A}$ & $-74.8(2)$ & $\mathrm{N} 8 \mathrm{~A}-\mathrm{C} 9 \mathrm{~A}-\mathrm{C} 10 \mathrm{~A}-\mathrm{C} 11 \mathrm{~A}$ & $52.82(18)$ \\
\hline $\mathrm{C} 11 \mathrm{~A}-\mathrm{N} 1 \mathrm{~A}-\mathrm{C} 2 \mathrm{~A}-\mathrm{C} 3 \mathrm{~A}$ & $108.53(17)$ & $\mathrm{C} 9 \mathrm{~A}-\mathrm{C} 10 \mathrm{~A}-\mathrm{C} 11 \mathrm{~A}-\mathrm{N} 1 \mathrm{~A}$ & $-52.69(19)$ \\
\hline $\mathrm{C} 2 \mathrm{~A}-\mathrm{N} 1 \mathrm{~A}-\mathrm{C} 7 \mathrm{~A}-\mathrm{N} 8 \mathrm{~A}$ & $-173.67(15)$ & $\mathrm{C} 6 \mathrm{~B}-\mathrm{C} 1 \mathrm{~B}-\mathrm{C} 2 \mathrm{~B}-\mathrm{C} 3 \mathrm{~B}$ & $1.0(2)$ \\
\hline $\mathrm{C} 2 \mathrm{~A}-\mathrm{N} 1 \mathrm{~A}-\mathrm{C} 7 \mathrm{~A}-\mathrm{C} 6 \mathrm{~A}$ & $10.2(2)$ & $\mathrm{C} 11 \mathrm{~B}-\mathrm{C} 1 \mathrm{~B}-\mathrm{C} 2 \mathrm{~B}-\mathrm{C} 3 \mathrm{~B}$ & $178.48(15)$ \\
\hline $\mathrm{C} 11 \mathrm{~A}-\mathrm{N} 1 \mathrm{~A}-\mathrm{C} 7 \mathrm{~A}-\mathrm{N} 8 \mathrm{~A}$ & $2.9(3)$ & $\mathrm{C} 2 \mathrm{~B}-\mathrm{C} 1 \mathrm{~B}-\mathrm{C} 6 \mathrm{~B}-\mathrm{C} 5 \mathrm{~B}$ & $0.1(2)$ \\
\hline $\mathrm{C} 11 \mathrm{~A}-\mathrm{N} 1 \mathrm{~A}-\mathrm{C} 7 \mathrm{~A}-\mathrm{C} 6 \mathrm{~A}$ & $-173.23(15)$ & $\mathrm{C} 11 \mathrm{~B}-\mathrm{C} 1 \mathrm{~B}-\mathrm{C} 6 \mathrm{~B}-\mathrm{C} 5 \mathrm{~B}$ & $-177.44(15)$ \\
\hline $\mathrm{C} 2 \mathrm{~A}-\mathrm{N} 1 \mathrm{~A}-\mathrm{C} 11 \mathrm{~A}-\mathrm{C} 10 \mathrm{~A}$ & $-157.91(14)$ & $\mathrm{C} 2 \mathrm{~B}-\mathrm{C} 1 \mathrm{~B}-\mathrm{C} 11 \mathrm{~B}-\mathrm{O} 11 \mathrm{~B}$ & $179.25(15)$ \\
\hline $\mathrm{C} 7 \mathrm{~A}-\mathrm{N} 1 \mathrm{~A}-\mathrm{C} 11 \mathrm{~A}-\mathrm{C} 10 \mathrm{~A}$ & $25.4(2)$ & $\mathrm{C} 2 \mathrm{~B}-\mathrm{C} 1 \mathrm{~B}-\mathrm{C} 11 \mathrm{~B}-\mathrm{O} 12 \mathrm{~B}$ & $-1.6(3)$ \\
\hline $\mathrm{C} 9 \mathrm{~A}-\mathrm{N} 8 \mathrm{~A}-\mathrm{C} 7 \mathrm{~A}-\mathrm{N} 1 \mathrm{~A}$ & $-2.2(3)$ & $\mathrm{C} 6 \mathrm{~B}-\mathrm{C} 1 \mathrm{~B}-\mathrm{C} 11 \mathrm{~B}-\mathrm{O} 11 \mathrm{~B}$ & $-3.4(2)$ \\
\hline $\mathrm{C} 9 \mathrm{~A}-\mathrm{N} 8 \mathrm{~A}-\mathrm{C} 7 \mathrm{~A}-\mathrm{C} 6 \mathrm{~A}$ & $174.06(15)$ & $\mathrm{C} 6 \mathrm{~B}-\mathrm{C} 1 \mathrm{~B}-\mathrm{C} 11 \mathrm{~B}-\mathrm{O} 12 \mathrm{~B}$ & $175.83(16)$ \\
\hline $\mathrm{C} 7 \mathrm{~A}-\mathrm{N} 8 \mathrm{~A}-\mathrm{C} 9 \mathrm{~A}-\mathrm{C} 10 \mathrm{~A}$ & $-26.7(2)$ & $\mathrm{C} 1 \mathrm{~B}-\mathrm{C} 2 \mathrm{~B}-\mathrm{C} 3 \mathrm{~B}-\mathrm{C} 4 \mathrm{~B}$ & $-0.9(2)$ \\
\hline $\mathrm{N} 1 \mathrm{~A}-\mathrm{C} 2 \mathrm{~A}-\mathrm{C} 3 \mathrm{~A}-\mathrm{C} 4 \mathrm{~A}$ & $77.87(18)$ & $\mathrm{C} 2 \mathrm{~B}-\mathrm{C} 3 \mathrm{~B}-\mathrm{C} 4 \mathrm{~B}-\mathrm{N} 4 \mathrm{~B}$ & $178.92(16)$ \\
\hline $\mathrm{C} 2 \mathrm{~A}-\mathrm{C} 3 \mathrm{~A}-\mathrm{C} 4 \mathrm{~A}-\mathrm{C} 5 \mathrm{~A}$ & $-56.71(19)$ & $\mathrm{C} 2 \mathrm{~B}-\mathrm{C} 3 \mathrm{~B}-\mathrm{C} 4 \mathrm{~B}-\mathrm{C} 5 \mathrm{~B}$ & $-0.4(2)$ \\
\hline $\mathrm{C} 3 \mathrm{~A}-\mathrm{C} 4 \mathrm{~A}-\mathrm{C} 5 \mathrm{~A}-\mathrm{C} 6 \mathrm{~A}$ & $62.97(19)$ & $\mathrm{N} 4 \mathrm{~B}-\mathrm{C} 4 \mathrm{~B}-\mathrm{C} 5 \mathrm{~B}-\mathrm{C} 6 \mathrm{~B}$ & $-177.86(16)$ \\
\hline $\mathrm{C} 4 \mathrm{~A}-\mathrm{C} 5 \mathrm{~A}-\mathrm{C} 6 \mathrm{~A}-\mathrm{C} 7 \mathrm{~A}$ & $-83.76(18)$ & $\mathrm{C} 3 \mathrm{~B}-\mathrm{C} 4 \mathrm{~B}-\mathrm{C} 5 \mathrm{~B}-\mathrm{C} 6 \mathrm{~B}$ & $1.4(2)$ \\
\hline $\mathrm{C} 5 \mathrm{~A}-\mathrm{C} 6 \mathrm{~A}-\mathrm{C} 7 \mathrm{~A}-\mathrm{N} 1 \mathrm{~A}$ & $60.9(2)$ & $\mathrm{C} 4 \mathrm{~B}-\mathrm{C} 5 \mathrm{~B}-\mathrm{C} 6 \mathrm{~B}-\mathrm{C} 1 \mathrm{~B}$ & $-1.3(3)$ \\
\hline $\mathrm{C} 5 \mathrm{~A}-\mathrm{C} 6 \mathrm{~A}-\mathrm{C} 7 \mathrm{~A}-\mathrm{N} 8 \mathrm{~A}$ & $-115.39(17)$ & & \\
\hline
\end{tabular}

Hydrogen-bond geometry $\left(A,{ }^{\circ}\right)$

\begin{tabular}{lllll}
\hline$D-\mathrm{H} \cdots A$ & $D-\mathrm{H}$ & $\mathrm{H} \cdots A$ & $D \cdots A$ & $D-\mathrm{H} \cdots A$ \\
\hline $\mathrm{N} 8 A-\mathrm{H} 8 A \cdots \mathrm{O} 11 B$ & $0.89(2)$ & $1.78(2)$ & $2.665(2)$ & $170(2)$ \\
$\mathrm{N} 4 B-\mathrm{H} 41 B \cdots \mathrm{O} 11 B^{\mathrm{i}}$ & $0.89(2)$ & $2.05(2)$ & $2.939(2)$ & $176(2)$ \\
$\mathrm{N} 4 B-\mathrm{H} 42 B \cdots \mathrm{O} 12 B^{\mathrm{ii}}$ & $0.92(2)$ & $1.98(2)$ & $2.891(2)$ & $176(2)$ \\
\hline
\end{tabular}

Symmetry codes: (i) $x+1 / 2,-y+1 / 2,-z+1$; (ii) $-x+1, y-1 / 2,-z+1 / 2$.

(II) Aza-8-azoniabicyclo[5.4.0] undec-7-ene 3,5-dinitrobenzoate

Crystal data

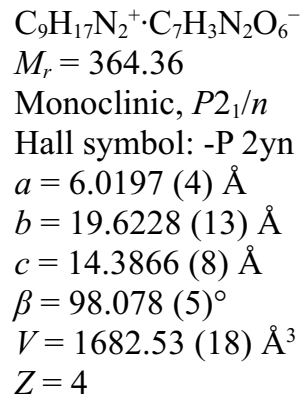

$F(000)=768$

$D_{\mathrm{x}}=1.438 \mathrm{Mg} \mathrm{m}^{-3}$

Mo $K \alpha$ radiation, $\lambda=0.71073 \AA$

Cell parameters from 1784 reflections

$\theta=4.0-28.0^{\circ}$

$\mu=0.11 \mathrm{~mm}^{-1}$

$T=200 \mathrm{~K}$

Needle, colourless

$0.30 \times 0.13 \times 0.08 \mathrm{~mm}$ 


\section{Data collection}

Oxford Diffraction Gemini-S CCD-detector diffractometer

Radiation source: fine-focus sealed tube Graphite monochromator

Detector resolution: 16.077 pixels $\mathrm{mm}^{-1}$ $\omega$ scans

Absorption correction: multi-scan

(CrysAlis PRO; Agilent, 2014)

$T_{\min }=0.90, T_{\max }=0.99$

Refinement

Refinement on $F^{2}$

Least-squares matrix: full

$R\left[F^{2}>2 \sigma\left(F^{2}\right)\right]=0.045$

$w R\left(F^{2}\right)=0.109$

$S=1.01$

3311 reflections

245 parameters

3 restraints

Primary atom site location: structure-invariant direct methods
7082 measured reflections

3311 independent reflections

2561 reflections with $I>2 \sigma(I)$

$R_{\text {int }}=0.024$

$\theta_{\max }=26.0^{\circ}, \theta_{\min }=3.4^{\circ}$

$h=-7 \rightarrow 7$

$k=-14 \longrightarrow 24$

$l=-9 \rightarrow 17$

Secondary atom site location: difference Fourier map

Hydrogen site location: inferred from neighbouring sites

$\mathrm{H}$ atoms treated by a mixture of independent and constrained refinement

$w=1 /\left[\sigma^{2}\left(F_{\mathrm{o}}^{2}\right)+(0.0435 P)^{2}+0.5615 P\right]$ where $P=\left(F_{\mathrm{o}}^{2}+2 F_{\mathrm{c}}{ }^{2}\right) / 3$

$(\Delta / \sigma)_{\max }<0.001$

$\Delta \rho_{\max }=0.18$ e $\AA^{-3}$

$\Delta \rho_{\min }=-0.22$ e $\AA^{-3}$

Special details

Geometry. Bond distances, angles etc. have been calculated using the rounded fractional coordinates. All su's are estimated from the variances of the (full) variance-covariance matrix. The cell esds are taken into account in the estimation of distances, angles and torsion angles

Refinement. Refinement of $\mathrm{F}^{2}$ against ALL reflections. The weighted R-factor $\mathrm{wR}$ and goodness of fit $\mathrm{S}$ are based on $\mathrm{F}^{2}$, conventional $R$-factors $R$ are based on $F$, with $F$ set to zero for negative $F^{2}$. The threshold expression of $F^{2}>2 \operatorname{sigma}\left(\mathrm{F}^{2}\right)$ is used only for calculating R-factors(gt) etc. and is not relevant to the choice of reflections for refinement. R-factors based on $\mathrm{F}^{2}$ are statistically about twice as large as those based on F, and R- factors based on ALL data will be even larger.

Fractional atomic coordinates and isotropic or equivalent isotropic displacement parameters $\left(\AA^{2}\right)$

\begin{tabular}{lllll}
\hline & $x$ & $y$ & $z$ & $U_{\text {iso }} * / U_{\text {eq }}$ \\
O11B & $-0.0061(2)$ & $0.68797(7)$ & $0.41185(9)$ & $0.0408(4)$ \\
O12B & $-0.0380(2)$ & $0.64765(8)$ & $0.26602(10)$ & $0.0556(5)$ \\
O31B & $-0.5921(3)$ & $0.46963(8)$ & $0.17213(10)$ & $0.0567(5)$ \\
O32B & $-0.8865(3)$ & $0.46500(9)$ & $0.24178(11)$ & $0.0703(6)$ \\
O51B & $-0.8471(2)$ & $0.55899(8)$ & $0.55381(10)$ & $0.0514(5)$ \\
O52B & $-0.5787(3)$ & $0.62813(8)$ & $0.60351(10)$ & $0.0576(6)$ \\
N3B & $-0.6966(3)$ & $0.48464(8)$ & $0.23584(11)$ & $0.0416(5)$ \\
N5B & $-0.6770(3)$ & $0.59011(8)$ & $0.54409(10)$ & $0.0363(5)$ \\
C1B & $-0.2967(3)$ & $0.60944(8)$ & $0.36270(11)$ & $0.0264(5)$ \\
C2B & $-0.3972(3)$ & $0.56537(8)$ & $0.29419(11)$ & $0.0288(5)$ \\
C3B & $-0.5892(3)$ & $0.53100(8)$ & $0.30888(11)$ & $0.0289(5)$ \\
C4B & $-0.6880(3)$ & $0.53844(8)$ & $0.38905(12)$ & $0.0293(5)$ \\
C5B & $-0.5807(3)$ & $0.58130(8)$ & $0.45649(11)$ & $0.0265(5)$ \\
C6B & $-0.3873(3)$ & $0.61637(8)$ & $0.44556(11)$ & $0.0269(5)$ \\
C11B & $-0.0952(3)$ & $0.65174(9)$ & $0.34516(13)$ & $0.0327(5)$ \\
N1A & $0.6514(2)$ & $0.81846(7)$ & $0.36517(9)$ & $0.0288(4)$
\end{tabular}




\begin{tabular}{|c|c|c|c|c|c|}
\hline N8A & $0.3270(3)$ & $0.75625(8)$ & $0.33371(10)$ & $0.0364(5)$ & \\
\hline $\mathrm{C} 2 \mathrm{~A}$ & $0.8281(3)$ & $0.85009(9)$ & $0.43221(13)$ & $0.0350(6)$ & \\
\hline $\mathrm{C} 3 \mathrm{~A}$ & $0.7557(3)$ & $0.91508(9)$ & $0.47621(13)$ & $0.0378(6)$ & \\
\hline $\mathrm{C} 4 \mathrm{~A}$ & $0.6172(3)$ & $0.90383(10)$ & $0.55531(13)$ & $0.0390(6)$ & \\
\hline $\mathrm{C} 5 \mathrm{~A}$ & $0.4046(3)$ & $0.86226(10)$ & $0.52884(13)$ & $0.0383(6)$ & \\
\hline C6A & $0.4433(3)$ & $0.78996(9)$ & $0.49381(11)$ & $0.0334(5)$ & \\
\hline C7A & $0.4773(3)$ & $0.78797(8)$ & $0.39270(11)$ & $0.0262(5)$ & \\
\hline C9A & $0.3565(6)$ & $0.74500(17)$ & $0.2353(2)$ & $0.0357(10)$ & $0.735(3)$ \\
\hline C10A & $0.4681(5)$ & $0.80757(15)$ & $0.20241(17)$ & $0.0364(8)$ & $0.735(3)$ \\
\hline C11A & 0.6839 & $0.82115(10)$ & $0.26593(12)$ & $0.0364(6)$ & \\
\hline C13A & $0.3000(16)$ & $0.7705(5)$ & $0.2305(8)$ & $0.0357(10)$ & $0.265(3)$ \\
\hline $\mathrm{C} 12 \mathrm{~A}$ & $0.5368(12)$ & $0.7669(4)$ & $0.2074(5)$ & $0.0364(8)$ & $0.265(3)$ \\
\hline $\mathrm{H} 2 \mathrm{~B}$ & -0.33470 & 0.55890 & 0.23780 & $0.0350 *$ & \\
\hline $\mathrm{H} 4 \mathrm{~B}$ & -0.82260 & 0.51530 & 0.39720 & $0.0350 *$ & \\
\hline H6B & -0.31700 & 0.64500 & 0.49430 & $0.0320 *$ & \\
\hline H8A & 0.217 & $0.7342(9)$ & $0.3570(12)$ & $0.0440 *$ & \\
\hline $\mathrm{H} 10 \mathrm{~A}$ & 0.49920 & 0.80070 & 0.13730 & $0.0440 *$ & $0.735(3)$ \\
\hline $\mathrm{H} 21 \mathrm{~A}$ & 0.95830 & 0.86030 & 0.39950 & $0.0420^{*}$ & \\
\hline $\mathrm{H} 22 \mathrm{~A}$ & 0.87810 & 0.81700 & 0.48270 & $0.0420 *$ & \\
\hline $\mathrm{H} 31 \mathrm{~A}$ & 0.66700 & 0.94280 & 0.42680 & $0.0450 *$ & \\
\hline $\mathrm{H} 32 \mathrm{~A}$ & 0.89130 & 0.94160 & 0.50080 & $0.0450^{*}$ & \\
\hline $\mathrm{H} 41 \mathrm{~A}$ & 0.57520 & 0.94880 & 0.57870 & $0.0470^{*}$ & \\
\hline $\mathrm{H} 42 \mathrm{~A}$ & 0.71200 & 0.88050 & 0.60760 & $0.0470 *$ & \\
\hline $\mathrm{H} 51 \mathrm{~A}$ & 0.30550 & 0.88690 & 0.47920 & $0.0460 *$ & \\
\hline $\mathrm{H} 52 \mathrm{~A}$ & 0.32480 & 0.85900 & 0.58440 & $0.0460^{*}$ & \\
\hline H61A & 0.57690 & 0.77030 & 0.53250 & $0.0400^{*}$ & \\
\hline $\mathrm{H} 62 \mathrm{~A}$ & 0.31250 & 0.76120 & 0.50230 & $0.0400 *$ & \\
\hline H91A & 0.20920 & 0.73760 & 0.19650 & $0.0430 *$ & $0.735(3)$ \\
\hline $\mathrm{H} 92 \mathrm{~A}$ & 0.45120 & 0.70430 & 0.22990 & $0.0430 *$ & $0.735(3)$ \\
\hline H11A & 0.36680 & 0.84730 & 0.20280 & $0.0440^{*}$ & $0.735(3)$ \\
\hline $\mathrm{H} 12 \mathrm{~A}$ & 0.79710 & 0.78680 & 0.25410 & $0.0440 *$ & $0.735(3)$ \\
\hline H13A & 0.74150 & 0.86670 & 0.25170 & $0.0440 *$ & $0.735(3)$ \\
\hline $\mathrm{H} 14 \mathrm{~A}$ & 0.53670 & 0.77550 & 0.13960 & $0.0440 *$ & $0.265(3)$ \\
\hline $\mathrm{H} 15 \mathrm{~A}$ & 0.59940 & 0.72090 & 0.22210 & $0.0440 *$ & $0.265(3)$ \\
\hline H16A & 0.23430 & 0.81620 & 0.21620 & $0.0430 *$ & $0.265(3)$ \\
\hline H17A & 0.20330 & 0.73590 & 0.19490 & $0.0430 *$ & $0.265(3)$ \\
\hline H18A & 0.84390 & 0.81290 & 0.26060 & $0.0440 *$ & $0.265(3)$ \\
\hline H19A & 0.64380 & 0.86710 & 0.24050 & $0.0440 *$ & $0.265(3)$ \\
\hline
\end{tabular}

Atomic displacement parameters $\left(\AA^{2}\right)$

\begin{tabular}{lllllll}
\hline & $U^{11}$ & $U^{22}$ & $U^{33}$ & $U^{12}$ & $U^{13}$ & $U^{23}$ \\
\hline O11B & $0.0376(7)$ & $0.0403(8)$ & $0.0454(7)$ & $-0.0139(6)$ & $0.0090(6)$ & $-0.0054(6)$ \\
O12B & $0.0558(9)$ & $0.0740(11)$ & $0.0419(8)$ & $-0.0258(8)$ & $0.0238(7)$ & $-0.0043(7)$ \\
O31B & $0.0690(10)$ & $0.0566(10)$ & $0.0423(8)$ & $0.0063(8)$ & $0.0003(7)$ & $-0.0213(7)$ \\
O32B & $0.0733(11)$ & $0.0811(12)$ & $0.0539(9)$ & $-0.0503(10)$ & $-0.0006(8)$ & $-0.0091(9)$ \\
O51B & $0.0472(8)$ & $0.0586(9)$ & $0.0543(8)$ & $-0.0128(7)$ & $0.0276(7)$ & $0.0036(7)$ \\
O52B & $0.0663(10)$ & $0.0729(11)$ & $0.0380(8)$ & $-0.0175(8)$ & $0.0228(7)$ & $-0.0229(8)$
\end{tabular}




\begin{tabular}{lllllll} 
& & & & \\
N3B & $0.0534(11)$ & $0.0353(9)$ & $0.0329(8)$ & $-0.0074(8)$ & $-0.0055(8)$ & $-0.0016(7)$ \\
N5B & $0.0392(9)$ & $0.0378(9)$ & $0.0343(8)$ & $-0.0006(7)$ & $0.0132(7)$ & $0.0021(7)$ \\
C1B & $0.0250(8)$ & $0.0242(8)$ & $0.0298(8)$ & $0.0011(7)$ & $0.0034(7)$ & $0.0032(7)$ \\
C2B & $0.0342(9)$ & $0.0280(9)$ & $0.0251(8)$ & $0.0037(7)$ & $0.0070(7)$ & $0.0017(7)$ \\
C3B & $0.0338(9)$ & $0.0244(9)$ & $0.0268(8)$ & $-0.0015(7)$ & $-0.0021(7)$ & $-0.0001(7)$ \\
C4B & $0.0263(8)$ & $0.0258(9)$ & $0.0352(9)$ & $-0.0016(7)$ & $0.0021(7)$ & $0.0055(8)$ \\
C5B & $0.0284(8)$ & $0.0259(9)$ & $0.0264(8)$ & $0.0035(7)$ & $0.0078(7)$ & $0.0013(7)$ \\
C6B & $0.0275(8)$ & $0.0247(9)$ & $0.0279(8)$ & $-0.0004(7)$ & $0.0020(7)$ & $-0.0018(7)$ \\
C11B & $0.0297(9)$ & $0.0309(9)$ & $0.0382(10)$ & $-0.0006(8)$ & $0.0072(8)$ & $0.0031(8)$ \\
N1A & $0.0255(7)$ & $0.0325(8)$ & $0.0285(7)$ & $-0.0044(6)$ & $0.0039(6)$ & $0.0020(6)$ \\
N8A & $0.0358(8)$ & $0.0484(10)$ & $0.0250(7)$ & $-0.0178(7)$ & $0.0045(6)$ & $-0.0008(7)$ \\
C2A & $0.0236(9)$ & $0.0388(10)$ & $0.0410(10)$ & $-0.0059(8)$ & $-0.0007(8)$ & $0.0001(8)$ \\
C3A & $0.0347(10)$ & $0.0330(10)$ & $0.0434(10)$ & $-0.0076(8)$ & $-0.0020(8)$ & $0.0000(9)$ \\
C4A & $0.0405(10)$ & $0.0399(11)$ & $0.0343(9)$ & $-0.0025(8)$ & $-0.0032(8)$ & $-0.0064(9)$ \\
C5A & $0.0357(10)$ & $0.0495(12)$ & $0.0302(9)$ & $-0.0046(9)$ & $0.0062(8)$ & $-0.0083(8)$ \\
C6A & $0.0357(10)$ & $0.0403(10)$ & $0.0237(8)$ & $-0.0113(8)$ & $0.0024(7)$ & $0.0050(8)$ \\
C7A & $0.0254(8)$ & $0.0250(8)$ & $0.0272(8)$ & $-0.0015(7)$ & $0.0006(7)$ & $0.0028(7)$ \\
C9A & $0.0418(19)$ & $0.041(2)$ & $0.0236(10)$ & $-0.0039(14)$ & $0.0026(12)$ & $-0.0018(16)$ \\
C10A & $0.0443(15)$ & $0.0390(16)$ & $0.0263(10)$ & $-0.0024(12)$ & $0.0061(10)$ & $0.0036(12)$ \\
C11A & $0.0348(10)$ & $0.0427(11)$ & $0.0339(9)$ & $-0.0043(8)$ & $0.0126(8)$ & $0.0047(8)$ \\
C13A & $0.0418(19)$ & $0.041(2)$ & $0.0236(10)$ & $-0.0039(14)$ & $0.0026(12)$ & $-0.0018(16)$ \\
C12A & $0.0443(15)$ & $0.0390(16)$ & $0.0263(10)$ & $-0.0024(12)$ & $0.0061(10)$ & $0.0036(12)$ \\
& & & & & & \\
\hline
\end{tabular}

Geometric parameters $\left(\AA,{ }^{\circ}\right)$

\begin{tabular}{llll}
\hline O11B-C11B & $1.253(2)$ & $\mathrm{C} 5 \mathrm{~A}-\mathrm{C} 6 \mathrm{~A}$ & $1.534(3)$ \\
$\mathrm{O} 12 \mathrm{~B}-\mathrm{C} 11 \mathrm{~B}$ & $1.238(2)$ & $\mathrm{C} 6 \mathrm{~A}-\mathrm{C} 7 \mathrm{~A}$ & $1.498(2)$ \\
$\mathrm{O} 31 \mathrm{~B}-\mathrm{N} 3 \mathrm{~B}$ & $1.218(2)$ & $\mathrm{C} 9 \mathrm{~A}-\mathrm{C} 10 \mathrm{~A}$ & $1.507(4)$ \\
$\mathrm{O} 32 \mathrm{~B}-\mathrm{N} 3 \mathrm{~B}$ & $1.221(3)$ & $\mathrm{C} 10 \mathrm{~A}-\mathrm{C} 11 \mathrm{~A}$ & $1.503(3)$ \\
$\mathrm{O} 51 \mathrm{~B}-\mathrm{N} 5 \mathrm{~B}$ & $1.217(2)$ & $\mathrm{C} 12 \mathrm{~A}-\mathrm{C} 13 \mathrm{~A}$ & $1.510(12)$ \\
$\mathrm{O} 52 \mathrm{~B}-\mathrm{N} 5 \mathrm{~B}$ & $1.223(2)$ & $\mathrm{C} 12 \mathrm{~A}-\mathrm{C} 11 \mathrm{~A}$ & $1.555(8)$ \\
$\mathrm{N} 3 \mathrm{~B}-\mathrm{C} 3 \mathrm{~B}$ & $1.469(2)$ & $\mathrm{C} 2 \mathrm{~A}-\mathrm{H} 21 \mathrm{~A}$ & 0.9900 \\
$\mathrm{~N} 5 \mathrm{~B}-\mathrm{C} 5 \mathrm{~B}$ & $1.470(2)$ & $\mathrm{C} 2 \mathrm{~A}-\mathrm{H} 22 \mathrm{~A}$ & 0.9900 \\
$\mathrm{~N} 1 \mathrm{~A}-\mathrm{C} 2 \mathrm{~A}$ & $1.469(2)$ & $\mathrm{C} 3 \mathrm{~A}-\mathrm{H} 31 \mathrm{~A}$ & 0.9900 \\
$\mathrm{~N} 1 \mathrm{~A}-\mathrm{C} 7 \mathrm{~A}$ & $1.315(2)$ & $\mathrm{C} 3 \mathrm{~A}-\mathrm{H} 32 \mathrm{~A}$ & 0.9900 \\
$\mathrm{~N} 1 \mathrm{~A}-\mathrm{C} 11 \mathrm{~A}$ & $1.469(2)$ & $\mathrm{C} 4 \mathrm{~A}-\mathrm{H} 41 \mathrm{~A}$ & 0.9900 \\
$\mathrm{~N} 8 \mathrm{~A}-\mathrm{C} 13 \mathrm{~A}$ & $1.497(11)$ & $\mathrm{C} 4 \mathrm{~A}-\mathrm{H} 42 \mathrm{~A}$ & 0.9900 \\
$\mathrm{~N} 8 \mathrm{~A}-\mathrm{C} 9 \mathrm{~A}$ & $1.468(3)$ & $\mathrm{C} 5 \mathrm{~A}-\mathrm{H} 51 \mathrm{~A}$ & 0.9900 \\
$\mathrm{~N} 8 \mathrm{~A}-\mathrm{C} 7 \mathrm{~A}$ & $1.308(2)$ & $\mathrm{C} 5 \mathrm{~A}-\mathrm{H} 52 \mathrm{~A}$ & 0.9900 \\
$\mathrm{~N} 8 \mathrm{~A}-\mathrm{H} 8 \mathrm{~A}$ & $0.895(18)$ & $\mathrm{C} 6 \mathrm{~A}-\mathrm{H} 61 \mathrm{~A}$ & 0.9900 \\
$\mathrm{C} 1 \mathrm{~B}-\mathrm{C} 11 \mathrm{~B}$ & $1.520(3)$ & $\mathrm{C} 6 \mathrm{~A}-\mathrm{H} 62 \mathrm{~A}$ & 0.9900 \\
$\mathrm{C} 1 \mathrm{~B}-\mathrm{C} 2 \mathrm{~B}$ & $1.385(2)$ & $\mathrm{C} 9 \mathrm{~A}-\mathrm{H} 91 \mathrm{~A}$ & 0.9900 \\
$\mathrm{C} 1 \mathrm{~B}-\mathrm{C} 6 \mathrm{~B}$ & $1.386(2)$ & $\mathrm{C} 9 \mathrm{~A}-\mathrm{H} 92 \mathrm{~A}$ & 0.9900 \\
$\mathrm{C} 2 \mathrm{~B}-\mathrm{C} 3 \mathrm{~B}$ & $1.380(2)$ & $\mathrm{C} 10 \mathrm{~A}-\mathrm{H} 10 \mathrm{~A}$ & 0.9900 \\
$\mathrm{C} 3 \mathrm{~B}-\mathrm{C} 4 \mathrm{~B}$ & $1.378(2)$ & $\mathrm{C} 10 \mathrm{~A}-\mathrm{H} 11 \mathrm{~A}$ & 0.9900 \\
$\mathrm{C} 4 \mathrm{~B}-\mathrm{C} 5 \mathrm{~B}$ & $1.375(2)$ & $\mathrm{C} 11 \mathrm{~A}-\mathrm{H} 12 \mathrm{~A}$ & 0.9900 \\
$\mathrm{C} 5 \mathrm{~B}-\mathrm{C} 6 \mathrm{~B}$ & $1.380(2)$ & $\mathrm{C} 11 \mathrm{~A}-\mathrm{H} 13 \mathrm{~A}$ & 0.9900 \\
$\mathrm{C} 2 \mathrm{~B}-\mathrm{H} 2 \mathrm{~B}$ & 0.9500 & $\mathrm{C} 12 \mathrm{~A}-\mathrm{H} 14 \mathrm{~A}$ & 0.9900
\end{tabular}




\begin{tabular}{|c|c|c|c|}
\hline $\mathrm{C} 4 \mathrm{~B}-\mathrm{H} 4 \mathrm{~B}$ & 0.9500 & $\mathrm{C} 12 \mathrm{~A}-\mathrm{H} 15 \mathrm{~A}$ & 0.9900 \\
\hline $\mathrm{C} 6 \mathrm{~B}-\mathrm{H} 6 \mathrm{~B}$ & 0.9500 & $\mathrm{C} 13 \mathrm{~A}-\mathrm{H} 16 \mathrm{~A}$ & 0.9900 \\
\hline $\mathrm{C} 2 \mathrm{~A}-\mathrm{C} 3 \mathrm{~A}$ & $1.515(3)$ & $\mathrm{C} 13 \mathrm{~A}-\mathrm{H} 17 \mathrm{~A}$ & 0.9900 \\
\hline $\mathrm{C} 3 \mathrm{~A}-\mathrm{C} 4 \mathrm{~A}$ & $1.518(3)$ & $\mathrm{C} 11 \mathrm{~A}-\mathrm{H} 18 \mathrm{~A}$ & 0.9900 \\
\hline $\mathrm{C} 4 \mathrm{~A}-\mathrm{C} 5 \mathrm{~A}$ & $1.520(3)$ & $\mathrm{C} 11 \mathrm{~A}-\mathrm{H} 19 \mathrm{~A}$ & 0.9900 \\
\hline $\mathrm{O} 31 \mathrm{~B}-\mathrm{N} 3 \mathrm{~B}-\mathrm{O} 32 \mathrm{~B}$ & $124.33(17)$ & $\mathrm{C} 3 \mathrm{~A}-\mathrm{C} 2 \mathrm{~A}-\mathrm{H} 22 \mathrm{~A}$ & 109.00 \\
\hline $\mathrm{O} 31 \mathrm{~B}-\mathrm{N} 3 \mathrm{~B}-\mathrm{C} 3 \mathrm{~B}$ & $117.77(17)$ & $\mathrm{H} 21 \mathrm{~A}-\mathrm{C} 2 \mathrm{~A}-\mathrm{H} 22 \mathrm{~A}$ & 108.00 \\
\hline $\mathrm{O} 32 \mathrm{~B}-\mathrm{N} 3 \mathrm{~B}-\mathrm{C} 3 \mathrm{~B}$ & $117.89(16)$ & $\mathrm{C} 2 \mathrm{~A}-\mathrm{C} 3 \mathrm{~A}-\mathrm{H} 31 \mathrm{~A}$ & 109.00 \\
\hline $\mathrm{O} 51 \mathrm{~B}-\mathrm{N} 5 \mathrm{~B}-\mathrm{O} 52 \mathrm{~B}$ & $123.92(16)$ & $\mathrm{C} 2 \mathrm{~A}-\mathrm{C} 3 \mathrm{~A}-\mathrm{H} 32 \mathrm{~A}$ & 109.00 \\
\hline $\mathrm{O} 51 \mathrm{~B}-\mathrm{N} 5 \mathrm{~B}-\mathrm{C} 5 \mathrm{~B}$ & $118.63(15)$ & $\mathrm{C} 4 \mathrm{~A}-\mathrm{C} 3 \mathrm{~A}-\mathrm{H} 31 \mathrm{~A}$ & 109.00 \\
\hline $\mathrm{O} 52 \mathrm{~B}-\mathrm{N} 5 \mathrm{~B}-\mathrm{C} 5 \mathrm{~B}$ & $117.44(16)$ & $\mathrm{C} 4 \mathrm{~A}-\mathrm{C} 3 \mathrm{~A}-\mathrm{H} 32 \mathrm{~A}$ & 109.00 \\
\hline $\mathrm{C} 2 \mathrm{~A}-\mathrm{N} 1 \mathrm{~A}-\mathrm{C} 11 \mathrm{~A}$ & $116.09(13)$ & $\mathrm{H} 31 \mathrm{~A}-\mathrm{C} 3 \mathrm{~A}-\mathrm{H} 32 \mathrm{~A}$ & 108.00 \\
\hline $\mathrm{C} 7 \mathrm{~A}-\mathrm{N} 1 \mathrm{~A}-\mathrm{C} 11 \mathrm{~A}$ & $121.98(14)$ & $\mathrm{C} 3 \mathrm{~A}-\mathrm{C} 4 \mathrm{~A}-\mathrm{H} 41 \mathrm{~A}$ & 109.00 \\
\hline $\mathrm{C} 2 \mathrm{~A}-\mathrm{N} 1 \mathrm{~A}-\mathrm{C} 7 \mathrm{~A}$ & $121.91(14)$ & $\mathrm{C} 3 \mathrm{~A}-\mathrm{C} 4 \mathrm{~A}-\mathrm{H} 42 \mathrm{~A}$ & 109.00 \\
\hline $\mathrm{C} 7 \mathrm{~A}-\mathrm{N} 8 \mathrm{~A}-\mathrm{C} 9 \mathrm{~A}$ & $122.1(2)$ & $\mathrm{C} 5 \mathrm{~A}-\mathrm{C} 4 \mathrm{~A}-\mathrm{H} 41 \mathrm{~A}$ & 108.00 \\
\hline $\mathrm{C} 7 \mathrm{~A}-\mathrm{N} 8 \mathrm{~A}-\mathrm{C} 13 \mathrm{~A}$ & $121.6(4)$ & $\mathrm{C} 5 \mathrm{~A}-\mathrm{C} 4 \mathrm{~A}-\mathrm{H} 42 \mathrm{~A}$ & 108.00 \\
\hline $\mathrm{C} 13 \mathrm{~A}-\mathrm{N} 8 \mathrm{~A}-\mathrm{H} 8 \mathrm{~A}$ & $118.6(12)$ & $\mathrm{H} 41 \mathrm{~A}-\mathrm{C} 4 \mathrm{~A}-\mathrm{H} 42 \mathrm{~A}$ & 108.00 \\
\hline $\mathrm{C} 9 \mathrm{~A}-\mathrm{N} 8 \mathrm{~A}-\mathrm{H} 8 \mathrm{~A}$ & $119.0(11)$ & $\mathrm{C} 4 \mathrm{~A}-\mathrm{C} 5 \mathrm{~A}-\mathrm{H} 51 \mathrm{~A}$ & 109.00 \\
\hline $\mathrm{C} 7 \mathrm{~A}-\mathrm{N} 8 \mathrm{~A}-\mathrm{H} 8 \mathrm{~A}$ & $117.9(11)$ & $\mathrm{C} 4 \mathrm{~A}-\mathrm{C} 5 \mathrm{~A}-\mathrm{H} 52 \mathrm{~A}$ & 109.00 \\
\hline $\mathrm{C} 2 \mathrm{~B}-\mathrm{C} 1 \mathrm{~B}-\mathrm{C} 6 \mathrm{~B}$ & $119.23(16)$ & $\mathrm{C} 6 \mathrm{~A}-\mathrm{C} 5 \mathrm{~A}-\mathrm{H} 51 \mathrm{~A}$ & 109.00 \\
\hline $\mathrm{C} 2 \mathrm{~B}-\mathrm{C} 1 \mathrm{~B}-\mathrm{C} 11 \mathrm{~B}$ & $120.13(15)$ & $\mathrm{C} 6 \mathrm{~A}-\mathrm{C} 5 \mathrm{~A}-\mathrm{H} 52 \mathrm{~A}$ & 109.00 \\
\hline $\mathrm{C} 6 \mathrm{~B}-\mathrm{C} 1 \mathrm{~B}-\mathrm{C} 11 \mathrm{~B}$ & $120.60(15)$ & $\mathrm{H} 51 \mathrm{~A}-\mathrm{C} 5 \mathrm{~A}-\mathrm{H} 52 \mathrm{~A}$ & 108.00 \\
\hline $\mathrm{C} 1 \mathrm{~B}-\mathrm{C} 2 \mathrm{~B}-\mathrm{C} 3 \mathrm{~B}$ & $119.19(15)$ & $\mathrm{C} 5 \mathrm{~A}-\mathrm{C} 6 \mathrm{~A}-\mathrm{H} 61 \mathrm{~A}$ & 109.00 \\
\hline $\mathrm{N} 3 \mathrm{~B}-\mathrm{C} 3 \mathrm{~B}-\mathrm{C} 2 \mathrm{~B}$ & $119.14(15)$ & $\mathrm{C} 5 \mathrm{~A}-\mathrm{C} 6 \mathrm{~A}-\mathrm{H} 62 \mathrm{~A}$ & 109.00 \\
\hline $\mathrm{N} 3 \mathrm{~B}-\mathrm{C} 3 \mathrm{~B}-\mathrm{C} 4 \mathrm{~B}$ & $117.78(16)$ & $\mathrm{C} 7 \mathrm{~A}-\mathrm{C} 6 \mathrm{~A}-\mathrm{H} 61 \mathrm{~A}$ & 109.00 \\
\hline $\mathrm{C} 2 \mathrm{~B}-\mathrm{C} 3 \mathrm{~B}-\mathrm{C} 4 \mathrm{~B}$ & $123.08(15)$ & $\mathrm{C} 7 \mathrm{~A}-\mathrm{C} 6 \mathrm{~A}-\mathrm{H} 62 \mathrm{~A}$ & 109.00 \\
\hline $\mathrm{C} 3 \mathrm{~B}-\mathrm{C} 4 \mathrm{~B}-\mathrm{C} 5 \mathrm{~B}$ & $116.13(16)$ & $\mathrm{H} 61 \mathrm{~A}-\mathrm{C} 6 \mathrm{~A}-\mathrm{H} 62 \mathrm{~A}$ & 108.00 \\
\hline $\mathrm{C} 4 \mathrm{~B}-\mathrm{C} 5 \mathrm{~B}-\mathrm{C} 6 \mathrm{~B}$ & $123.01(16)$ & N8A-C9A-H91A & 110.00 \\
\hline $\mathrm{N} 5 \mathrm{~B}-\mathrm{C} 5 \mathrm{~B}-\mathrm{C} 4 \mathrm{~B}$ & $118.32(16)$ & $\mathrm{N} 8 \mathrm{~A}-\mathrm{C} 9 \mathrm{~A}-\mathrm{H} 92 \mathrm{~A}$ & 110.00 \\
\hline $\mathrm{N} 5 \mathrm{~B}-\mathrm{C} 5 \mathrm{~B}-\mathrm{C} 6 \mathrm{~B}$ & $118.67(14)$ & $\mathrm{C} 10 \mathrm{~A}-\mathrm{C} 9 \mathrm{~A}-\mathrm{H} 91 \mathrm{~A}$ & 110.00 \\
\hline $\mathrm{C} 1 \mathrm{~B}-\mathrm{C} 6 \mathrm{~B}-\mathrm{C} 5 \mathrm{~B}$ & $119.30(15)$ & $\mathrm{C} 10 \mathrm{~A}-\mathrm{C} 9 \mathrm{~A}-\mathrm{H} 92 \mathrm{~A}$ & 110.00 \\
\hline $\mathrm{O} 11 \mathrm{~B}-\mathrm{C} 11 \mathrm{~B}-\mathrm{C} 1 \mathrm{~B}$ & $116.66(16)$ & $\mathrm{H} 91 \mathrm{~A}-\mathrm{C} 9 \mathrm{~A}-\mathrm{H} 92 \mathrm{~A}$ & 108.00 \\
\hline $\mathrm{O} 11 \mathrm{~B}-\mathrm{C} 11 \mathrm{~B}-\mathrm{O} 12 \mathrm{~B}$ & $126.65(17)$ & $\mathrm{C} 9 \mathrm{~A}-\mathrm{C} 10 \mathrm{~A}-\mathrm{H} 10 \mathrm{~A}$ & 110.00 \\
\hline $\mathrm{O} 12 \mathrm{~B}-\mathrm{C} 11 \mathrm{~B}-\mathrm{C} 1 \mathrm{~B}$ & $116.68(16)$ & $\mathrm{C} 9 \mathrm{~A}-\mathrm{C} 10 \mathrm{~A}-\mathrm{H} 11 \mathrm{~A}$ & 110.00 \\
\hline $\mathrm{C} 3 \mathrm{~B}-\mathrm{C} 2 \mathrm{~B}-\mathrm{H} 2 \mathrm{~B}$ & 120.00 & $\mathrm{C} 11 \mathrm{~A}-\mathrm{C} 10 \mathrm{~A}-\mathrm{H} 10 \mathrm{~A}$ & 110.00 \\
\hline $\mathrm{C} 1 \mathrm{~B}-\mathrm{C} 2 \mathrm{~B}-\mathrm{H} 2 \mathrm{~B}$ & 120.00 & $\mathrm{C} 11 \mathrm{~A}-\mathrm{C} 10 \mathrm{~A}-\mathrm{H} 11 \mathrm{~A}$ & 110.00 \\
\hline $\mathrm{C} 3 \mathrm{~B}-\mathrm{C} 4 \mathrm{~B}-\mathrm{H} 4 \mathrm{~B}$ & 122.00 & $\mathrm{H} 10 \mathrm{~A}-\mathrm{C} 10 \mathrm{~A}-\mathrm{H} 11 \mathrm{~A}$ & 108.00 \\
\hline $\mathrm{C} 5 \mathrm{~B}-\mathrm{C} 4 \mathrm{~B}-\mathrm{H} 4 \mathrm{~B}$ & 122.00 & $\mathrm{~N} 1 \mathrm{~A}-\mathrm{C} 11 \mathrm{~A}-\mathrm{H} 12 \mathrm{~A}$ & 109.00 \\
\hline $\mathrm{C} 5 \mathrm{~B}-\mathrm{C} 6 \mathrm{~B}-\mathrm{H} 6 \mathrm{~B}$ & 120.00 & $\mathrm{~N} 1 \mathrm{~A}-\mathrm{C} 11 \mathrm{~A}-\mathrm{H} 13 \mathrm{~A}$ & 109.00 \\
\hline $\mathrm{C} 1 \mathrm{~B}-\mathrm{C} 6 \mathrm{~B}-\mathrm{H} 6 \mathrm{~B}$ & 120.00 & $\mathrm{C} 10 \mathrm{~A}-\mathrm{C} 11 \mathrm{~A}-\mathrm{H} 12 \mathrm{~A}$ & 109.00 \\
\hline $\mathrm{N} 1 \mathrm{~A}-\mathrm{C} 2 \mathrm{~A}-\mathrm{C} 3 \mathrm{~A}$ & $113.94(15)$ & $\mathrm{C} 10 \mathrm{~A}-\mathrm{C} 11 \mathrm{~A}-\mathrm{H} 13 \mathrm{~A}$ & 109.00 \\
\hline $\mathrm{C} 2 \mathrm{~A}-\mathrm{C} 3 \mathrm{~A}-\mathrm{C} 4 \mathrm{~A}$ & $114.29(15)$ & $\mathrm{H} 12 \mathrm{~A}-\mathrm{C} 11 \mathrm{~A}-\mathrm{H} 13 \mathrm{~A}$ & 108.00 \\
\hline $\mathrm{C} 3 \mathrm{~A}-\mathrm{C} 4 \mathrm{~A}-\mathrm{C} 5 \mathrm{~A}$ & $114.95(15)$ & $\mathrm{C} 13 \mathrm{~A}-\mathrm{C} 12 \mathrm{~A}-\mathrm{H} 14 \mathrm{~A}$ & 110.00 \\
\hline $\mathrm{C} 4 \mathrm{~A}-\mathrm{C} 5 \mathrm{~A}-\mathrm{C} 6 \mathrm{~A}$ & $114.65(15)$ & $\mathrm{C} 13 \mathrm{~A}-\mathrm{C} 12 \mathrm{~A}-\mathrm{H} 15 \mathrm{~A}$ & 110.00 \\
\hline $\mathrm{C} 5 \mathrm{~A}-\mathrm{C} 6 \mathrm{~A}-\mathrm{C} 7 \mathrm{~A}$ & $113.01(14)$ & $\mathrm{H} 14 \mathrm{~A}-\mathrm{C} 12 \mathrm{~A}-\mathrm{H} 15 \mathrm{~A}$ & 108.00 \\
\hline $\mathrm{N} 1 \mathrm{~A}-\mathrm{C} 7 \mathrm{~A}-\mathrm{N} 8 \mathrm{~A}$ & $121.94(15)$ & $\mathrm{N} 8 \mathrm{~A}-\mathrm{C} 13 \mathrm{~A}-\mathrm{H} 16 \mathrm{~A}$ & 111.00 \\
\hline $\mathrm{N} 1 \mathrm{~A}-\mathrm{C} 7 \mathrm{~A}-\mathrm{C} 6 \mathrm{~A}$ & $120.27(15)$ & $\mathrm{N} 8 \mathrm{~A}-\mathrm{C} 13 \mathrm{~A}-\mathrm{H} 17 \mathrm{~A}$ & 111.00 \\
\hline
\end{tabular}




$\begin{array}{ll}\mathrm{N} 8 \mathrm{~A}-\mathrm{C} 7 \mathrm{~A}-\mathrm{C} 6 \mathrm{~A} & 117.79(16) \\ \mathrm{N} 8 \mathrm{~A}-\mathrm{C} 9 \mathrm{~A}-\mathrm{C} 10 \mathrm{~A} & 107.5(2) \\ \mathrm{C} 9 \mathrm{~A}-\mathrm{C} 10 \mathrm{~A}-\mathrm{C} 11 \mathrm{~A} & 109.8(2) \\ \mathrm{N} 1 \mathrm{~A}-\mathrm{C} 11 \mathrm{~A}-\mathrm{C} 10 \mathrm{~A} & 11.27(16) \\ \mathrm{N} 8 \mathrm{~A}-\mathrm{C} 13 \mathrm{~A}-\mathrm{C} 12 \mathrm{~A} & 103.6(7) \\ \mathrm{N} 1 \mathrm{~A}-\mathrm{C} 2 \mathrm{~A}-\mathrm{H} 21 \mathrm{~A} & 109.00 \\ \mathrm{~N} 1 \mathrm{~A}-\mathrm{C} 2 \mathrm{~A}-\mathrm{H} 22 \mathrm{~A} & 109.00 \\ \mathrm{C} 3 \mathrm{~A}-\mathrm{C} 2 \mathrm{~A}-\mathrm{H} 21 \mathrm{~A} & 109.00 \\ & \\ \text { O31B-N3B-C3B-C2B } & 12.0(2) \\ \text { O31B-N3B-C3B-C4B } & -168.62(16) \\ \text { O32B-N3B-C3B-C2B } & -166.31(17) \\ \text { O32B-N3B-C3B-C4B } & 13.1(2) \\ \text { O51B-N5B-C5B-C4B } & 0.3(2) \\ \text { O51B-N5B-C5B-C6B } & -179.75(16) \\ \text { O52B-N5B-C5B-C4B } & 179.61(16) \\ \text { O52B-N5B-C5B-C6B } & -0.5(2) \\ \text { C2A-N1A-C11A-C10A } & -162.56(17) \\ \text { C7A-N1A-C2A-C3A } & -71.6(2) \\ \text { C11A-N1A-C2A-C3A } & 110.16(17) \\ \text { C2A-N1A-C7A-N8A } & -175.79(16) \\ \text { C2A-N1A-C7A-C6A } & 5.5(2) \\ \text { C11A-N1A-C7A-N8A } & 2.4(3) \\ \text { C11A-N1A-C7A-C6A } & -176.35(15) \\ \text { C7A-N1A-C11A-C10A } & 19.2(2) \\ \text { C9A-N8A-C7A-C6A } & -173.3(2) \\ \text { C9A-N8A-C7A-N1A } & 7.9(3) \\ \text { C7A-N8A-C9A-C10A } & -37.5(3) \\ \text { C6B-C1B-C2B-C3B } & 2.0(2) \\ \text { C2B-C1B-C11B-O11B } & -176.60(16) \\ \text { C2B-C1B-C11B-O12B } & 4.4(2) \\ & \end{array}$

$$
\begin{aligned}
& \text { C12A-C13A-H16A } \\
& \text { C12A-C13A-H17A } \\
& \text { H16A-C13A-H17A } \\
& \text { N1A-C11A-H18A } \\
& \text { N1A-C11A-H19A } \\
& \text { C12A-C11A-H18A } \\
& \text { C12A-C11A-H19A } \\
& \text { H18A-C11A-H19A }
\end{aligned}
$$$$
\mathrm{C} 6 \mathrm{~B}-\mathrm{C} 1 \mathrm{~B}-\mathrm{C} 11 \mathrm{~B}-\mathrm{O} 11 \mathrm{~B} \quad 5.9(2)
$$$$
\mathrm{C} 6 \mathrm{~B}-\mathrm{C} 1 \mathrm{~B}-\mathrm{C} 11 \mathrm{~B}-\mathrm{O} 12 \mathrm{~B} \quad-173.03(16)
$$$$
\mathrm{C} 11 \mathrm{~B}-\mathrm{C} 1 \mathrm{~B}-\mathrm{C} 2 \mathrm{~B}-\mathrm{C} 3 \mathrm{~B} \quad-175.52(15)
$$$$
\mathrm{C} 2 \mathrm{~B}-\mathrm{C} 1 \mathrm{~B}-\mathrm{C} 6 \mathrm{~B}-\mathrm{C} 5 \mathrm{~B} \quad-2.6(2)
$$$$
\mathrm{C} 11 \mathrm{~B}-\mathrm{C} 1 \mathrm{~B}-\mathrm{C} 6 \mathrm{~B}-\mathrm{C} 5 \mathrm{~B} \quad 174.94 \text { (15) }
$$$$
\mathrm{C} 1 \mathrm{~B}-\mathrm{C} 2 \mathrm{~B}-\mathrm{C} 3 \mathrm{~B}-\mathrm{N} 3 \mathrm{~B} \quad 179.56(15)
$$$$
\mathrm{C} 1 \mathrm{~B}-\mathrm{C} 2 \mathrm{~B}-\mathrm{C} 3 \mathrm{~B}-\mathrm{C} 4 \mathrm{~B} \quad 0.2(3)
$$$$
\mathrm{C} 2 \mathrm{~B}-\mathrm{C} 3 \mathrm{~B}-\mathrm{C} 4 \mathrm{~B}-\mathrm{C} 5 \mathrm{~B} \quad-1.7 \text { (2) }
$$$$
\mathrm{N} 3 \mathrm{~B}-\mathrm{C} 3 \mathrm{~B}-\mathrm{C} 4 \mathrm{~B}-\mathrm{C} 5 \mathrm{~B} \quad 178.91 \text { (15) }
$$$$
\mathrm{C} 3 \mathrm{~B}-\mathrm{C} 4 \mathrm{~B}-\mathrm{C} 5 \mathrm{~B}-\mathrm{C} 6 \mathrm{~B} \quad 1.1(2)
$$$$
\mathrm{C} 3 \mathrm{~B}-\mathrm{C} 4 \mathrm{~B}-\mathrm{C} 5 \mathrm{~B}-\mathrm{N} 5 \mathrm{~B} \quad-178.96(15)
$$$$
\mathrm{N} 5 \mathrm{~B}-\mathrm{C} 5 \mathrm{~B}-\mathrm{C} 6 \mathrm{~B}-\mathrm{C} 1 \mathrm{~B} \quad-178.93(15)
$$$$
\mathrm{C} 4 \mathrm{~B}-\mathrm{C} 5 \mathrm{~B}-\mathrm{C} 6 \mathrm{~B}-\mathrm{C} 1 \mathrm{~B} \quad 1.0 \text { (3) }
$$$$
\mathrm{N} 1 \mathrm{~A}-\mathrm{C} 2 \mathrm{~A}-\mathrm{C} 3 \mathrm{~A}-\mathrm{C} 4 \mathrm{~A} \quad 78.97(19)
$$$$
\mathrm{C} 2 \mathrm{~A}-\mathrm{C} 3 \mathrm{~A}-\mathrm{C} 4 \mathrm{~A}-\mathrm{C} 5 \mathrm{~A} \quad-57.1(2)
$$$$
\mathrm{C} 3 \mathrm{~A}-\mathrm{C} 4 \mathrm{~A}-\mathrm{C} 5 \mathrm{~A}-\mathrm{C} 6 \mathrm{~A} \quad 60.0(2)
$$$$
\mathrm{C} 4 \mathrm{~A}-\mathrm{C} 5 \mathrm{~A}-\mathrm{C} 6 \mathrm{~A}-\mathrm{C} 7 \mathrm{~A} \quad-81.00(19)
$$$$
\mathrm{C} 5 \mathrm{~A}-\mathrm{C} 6 \mathrm{~A}-\mathrm{C} 7 \mathrm{~A}-\mathrm{N} 1 \mathrm{~A} \quad 63.5(2)
$$$$
\mathrm{C} 5 \mathrm{~A}-\mathrm{C} 6 \mathrm{~A}-\mathrm{C} 7 \mathrm{~A}-\mathrm{N} 8 \mathrm{~A} \quad-115.29 \text { (18) }
$$$$
\mathrm{N} 8 \mathrm{~A}-\mathrm{C} 9 \mathrm{~A}-\mathrm{C} 10 \mathrm{~A}-\mathrm{C} 11 \mathrm{~A} \quad 55.9(3)
$$$$
\mathrm{C} 9 \mathrm{~A}-\mathrm{C} 10 \mathrm{~A}-\mathrm{C} 11 \mathrm{~A}-\mathrm{N} 1 \mathrm{~A} \quad-48.3 \text { (3) }
$$

Hydrogen-bond geometry $\left(\AA,{ }^{\circ}\right)$

\begin{tabular}{lllll}
\hline$D-\mathrm{H} \cdots A$ & $D-\mathrm{H}$ & $\mathrm{H} \cdots A$ & $D \cdots A$ & $D-\mathrm{H} \cdots A$ \\
\hline $\mathrm{N} 8 A-\mathrm{H} 8 A \cdots \mathrm{O} 11 B$ & $0.90(2)$ & $1.88(2)$ & $2.777(2)$ & $177(2)$ \\
$\mathrm{N} 8 A-\mathrm{H} 8 A \cdots \mathrm{O} 12 B$ & $0.90(2)$ & $2.53(2)$ & $3.117(2)$ & $124(1)$ \\
$\mathrm{C} 10 A-\mathrm{H} 11 A \cdots \mathrm{O} 32 B^{\mathrm{i}}$ & 0.99 & 2.44 & $3.247(3)$ & 138 \\
$\mathrm{C} 11 A-\mathrm{H} 13 A \cdots \mathrm{O} 52 B^{\mathrm{ii}}$ & 0.99 & 2.52 & $3.071(2)$ & 115 \\
$\mathrm{C} 2 A-\mathrm{H} 21 A \cdots \mathrm{O} 31 B^{\text {iii }}$ & 0.99 & 2.56 & $3.309(2)$ & 133 \\
$\mathrm{C} 6 A-\mathrm{H} 62 A \cdots \mathrm{O} 11 B$ & 0.99 & 2.60 & $3.438(2)$ & 143 \\
$\mathrm{C} 9 A-\mathrm{H} 91 A \cdots \mathrm{O} 12 B$ & 0.99 & 2.60 & $3.127(4)$ & 114 \\
\hline
\end{tabular}

Symmetry codes: (i) $-x-1 / 2, y+1 / 2,-z+1 / 2$; (ii) $x+3 / 2,-y+3 / 2, z-1 / 2$; (iii) $-x+1 / 2, y+1 / 2,-z+1 / 2$. 
(III) 1-Aza-8-azoniabicyclo[5.4.0] undec-7-ene 2-hydroxy-3,5-dinitrobenzoate

Crystal data

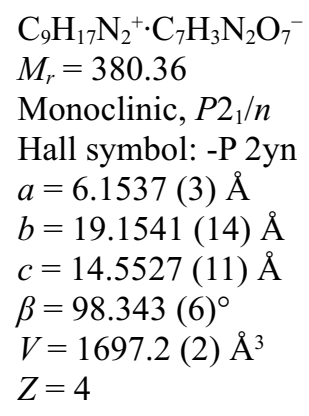

\section{Data collection}

Oxford Diffraction Gemini-S CCD-detector diffractometer

Radiation source: Enhance (Mo) X-ray source Graphite monochromator

Detector resolution: 16.077 pixels $\mathrm{mm}^{-1}$

$\omega$ scans

Absorption correction: multi-scan

(CrysAlis PRO; Agilent, 2014)

$T_{\min }=0.920, T_{\max }=0.990$

\section{Refinement}

Refinement on $F^{2}$

Least-squares matrix: full

$R\left[F^{2}>2 \sigma\left(F^{2}\right)\right]=0.058$

$w R\left(F^{2}\right)=0.123$

$S=1.03$

3339 reflections

263 parameters

3 restraints

Primary atom site location: structure-invariant direct methods
$F(000)=800$

$D_{\mathrm{x}}=1.489 \mathrm{Mg} \mathrm{m}^{-3}$

Mo $K \alpha$ radiation, $\lambda=0.71073 \AA$

Cell parameters from 1891 reflections

$\theta=3.5-26.6^{\circ}$

$\mu=0.12 \mathrm{~mm}^{-1}$

$T=200 \mathrm{~K}$

Needle, yellow

$0.30 \times 0.13 \times 0.10 \mathrm{~mm}$

7800 measured reflections

3339 independent reflections

2347 reflections with $I>2 \sigma(I)$

$R_{\text {int }}=0.034$

$\theta_{\max }=26.0^{\circ}, \theta_{\min }=3.4^{\circ}$

$h=-7 \rightarrow 7$

$k=-23 \rightarrow 23$

$l=-17 \rightarrow 17$

Secondary atom site location: difference Fourier map

Hydrogen site location: inferred from neighbouring sites

$\mathrm{H}$ atoms treated by a mixture of independent and constrained refinement

$w=1 /\left[\sigma^{2}\left(F_{\mathrm{o}}^{2}\right)+(0.0374 P)^{2}+0.7569 P\right]$ where $P=\left(F_{\mathrm{o}}^{2}+2 F_{\mathrm{c}}^{2}\right) / 3$

$(\Delta / \sigma)_{\max }<0.001$

$\Delta \rho_{\max }=0.29$ e $\AA^{-3}$

$\Delta \rho_{\min }=-0.29$ e $\AA^{-3}$

\section{Special details}

Geometry. Bond distances, angles etc. have been calculated using the rounded fractional coordinates. All su's are estimated from the variances of the (full) variance-covariance matrix. The cell esds are taken into account in the estimation of distances, angles and torsion angles

Refinement. Refinement of $\mathrm{F}^{2}$ against ALL reflections. The weighted R-factor $\mathrm{wR}$ and goodness of fit $\mathrm{S}$ are based on $\mathrm{F}^{2}$, conventional R-factors $R$ are based on $F$, with $F$ set to zero for negative $F^{2}$. The threshold expression of $F^{2}>2 \operatorname{sigma}\left(F^{2}\right)$ is used only for calculating R-factors(gt) etc. and is not relevant to the choice of reflections for refinement. R-factors based on $\mathrm{F}^{2}$ are statistically about twice as large as those based on $\mathrm{F}$, and R- factors based on ALL data will be even larger.

Fractional atomic coordinates and isotropic or equivalent isotropic displacement parameters $\left(\AA^{2}\right)$

\begin{tabular}{llllll}
\hline & $x$ & $y$ & $z$ & $U_{\text {iso }} * / U_{\text {eq }}$ & Occ. $(<1)$ \\
\hline O2B & $0.8426(4)$ & $0.56153(13)$ & $0.78929(15)$ & $0.0433(8)$ & 0.720 \\
O11B & $0.5084(3)$ & $0.68879(9)$ & $0.59293(13)$ & $0.0433(6)$ & \\
O12B & $0.5450(3)$ & $0.64525(10)$ & $0.73596(13)$ & $0.0522(7)$ & \\
O31B & $1.1116(4)$ & $0.45700(12)$ & $0.81707(17)$ & $0.0819(10)$ &
\end{tabular}




\begin{tabular}{|c|c|c|c|c|c|}
\hline $\mathrm{O} 32 \mathrm{~B}$ & $1.4080(4)$ & $0.47261(13)$ & $0.75765(15)$ & $0.0761(9)$ & \\
\hline O51B & $1.3286(3)$ & $0.55867(11)$ & $0.44585(14)$ & $0.0594(7)$ & \\
\hline O52B & $1.0707(4)$ & $0.63206(11)$ & $0.39870(14)$ & $0.0670(8)$ & \\
\hline N3B & $1.2118(4)$ & $0.48306(12)$ & $0.76028(16)$ & $0.0467(8)$ & \\
\hline N5B & $1.1654(3)$ & $0.59169(11)$ & $0.45698(15)$ & $0.0407(7)$ & \\
\hline $\mathrm{C} 1 \mathrm{~B}$ & $0.8002(3)$ & $0.60950(11)$ & $0.63899(16)$ & $0.0268(7)$ & \\
\hline $\mathrm{C} 2 \mathrm{~B}$ & $0.9062(3)$ & $0.56600(12)$ & $0.70947(16)$ & $0.0297(7)$ & \\
\hline $\mathrm{C} 3 \mathrm{~B}$ & $1.0956(4)$ & $0.53052(12)$ & $0.69146(16)$ & $0.0310(7)$ & \\
\hline $\mathrm{C} 4 \mathrm{~B}$ & $1.1816(3)$ & $0.53943(11)$ & $0.61041(16)$ & $0.0308(7)$ & \\
\hline $\mathrm{C} 5 \mathrm{~B}$ & $1.0735(3)$ & $0.58226(11)$ & $0.54278(15)$ & $0.0276(7)$ & \\
\hline $\mathrm{C} 6 \mathrm{~B}$ & $0.8810(3)$ & $0.61671(11)$ & $0.55531(15)$ & $0.0263(7)$ & \\
\hline $\mathrm{C} 11 \mathrm{~B}$ & $0.6029(4)$ & $0.65080(12)$ & 0.65595 (19) & $0.0346(8)$ & \\
\hline $\mathrm{O} 21 \mathrm{~B}$ & $0.7762(10)$ & $0.6571(3)$ & $0.4915(5)$ & $0.052(3)$ & 0.280 \\
\hline N1A & $-0.1524(3)$ & $0.82026(10)$ & $0.63820(13)$ & $0.0293(6)$ & \\
\hline N8A & $0.1714(3)$ & $0.76040(11)$ & $0.67301(14)$ & $0.0369(7)$ & \\
\hline $\mathrm{C} 2 \mathrm{~A}$ & $-0.3262(3)$ & $0.85087(13)$ & $0.56984(17)$ & $0.0357(8)$ & \\
\hline $\mathrm{C} 3 \mathrm{~A}$ & $-0.2606(4)$ & $0.91805(13)$ & $0.52684(18)$ & $0.0397(8)$ & \\
\hline $\mathrm{C} 4 \mathrm{~A}$ & $-0.1188(4)$ & $0.90797(14)$ & $0.45044(17)$ & $0.0409(8)$ & \\
\hline $\mathrm{C} 5 \mathrm{~A}$ & $0.0934(4)$ & $0.86814(13)$ & $0.48033(17)$ & $0.0393(9)$ & \\
\hline C6A & $0.0612(4)$ & $0.79368(13)$ & $0.51409(16)$ & $0.0340(8)$ & \\
\hline C7A & $0.0226(3)$ & $0.79083(11)$ & $0.61294(15)$ & $0.0265(7)$ & \\
\hline C9A & $0.1399(9)$ & $0.7478(2)$ & $0.7696(4)$ & $0.0366(18)$ & $0.686(4)$ \\
\hline $\mathrm{C} 10 \mathrm{~A}$ & $0.0234(6)$ & $0.8111(2)$ & $0.8005(3)$ & $0.0379(11)$ & $0.686(4)$ \\
\hline $\mathrm{C} 11 \mathrm{~A}$ & $-0.1871(4)$ & $0.82349(13)$ & $0.73612(16)$ & $0.0363(8)$ & \\
\hline $\mathrm{C} 13 \mathrm{~A}$ & $0.189(2)$ & $0.7738(7)$ & $0.7752(11)$ & $0.0366(18)$ & $0.314(4)$ \\
\hline $\mathrm{C} 12 \mathrm{~A}$ & $-0.0464(13)$ & $0.7704(5)$ & $0.7958(6)$ & $0.0379(11)$ & 0.314 (4) \\
\hline $\mathrm{H} 4 \mathrm{~B}$ & 1.31350 & 0.51650 & 0.60110 & $0.0370 *$ & \\
\hline H6B & 0.80240 & 0.64380 & 0.50700 & $0.0320^{*}$ & 0.720 \\
\hline $\mathrm{H} 2 \mathrm{~B}$ & 0.73870 & 0.58950 & 0.79190 & $0.0650^{*}$ & 0.720 \\
\hline H21B & 0.66080 & 0.67200 & 0.50930 & $0.0770^{*}$ & 0.280 \\
\hline H61B & 0.85460 & 0.56120 & 0.76770 & $0.0360^{*}$ & 0.280 \\
\hline $\mathrm{H} 8 \mathrm{~A}$ & $0.280(3)$ & $0.7394(11)$ & $0.6508(15)$ & $0.0320^{*}$ & \\
\hline $\mathrm{H} 10 \mathrm{~A}$ & -0.00890 & 0.80380 & 0.86450 & $0.0460^{*}$ & $0.686(4)$ \\
\hline $\mathrm{H} 21 \mathrm{~A}$ & -0.45670 & 0.85990 & 0.60060 & $0.0430^{*}$ & \\
\hline $\mathrm{H} 22 \mathrm{~A}$ & -0.36910 & 0.81640 & 0.51980 & $0.0430^{*}$ & \\
\hline H31A & -0.17940 & 0.94750 & 0.57630 & $0.0480^{*}$ & \\
\hline $\mathrm{H} 32 \mathrm{~A}$ & -0.39530 & 0.94360 & 0.50080 & $0.0480^{*}$ & \\
\hline $\mathrm{H} 41 \mathrm{~A}$ & -0.20570 & 0.88270 & 0.39820 & $0.0490^{*}$ & \\
\hline $\mathrm{H} 42 \mathrm{~A}$ & -0.08210 & 0.95440 & 0.42720 & $0.0490^{*}$ & \\
\hline H51A & 0.18300 & 0.89440 & 0.53080 & $0.0470^{*}$ & \\
\hline H52A & 0.17720 & 0.86610 & 0.42730 & $0.0470^{*}$ & \\
\hline H61A & -0.06570 & 0.77230 & 0.47440 & $0.0410^{*}$ & \\
\hline H62A & 0.19310 & 0.76570 & 0.50720 & $0.0410^{*}$ & \\
\hline H91A & 0.28350 & 0.74140 & 0.80920 & $0.0440^{*}$ & $0.686(4)$ \\
\hline H92A & 0.05040 & 0.70540 & 0.77390 & $0.0440^{*}$ & $0.686(4)$ \\
\hline H11A & 0.11950 & 0.85260 & 0.80070 & $0.0460^{*}$ & $0.686(4)$ \\
\hline $\mathrm{H} 12 \mathrm{~A}$ & -0.29640 & 0.78780 & 0.74760 & $0.0440^{*}$ & $0.686(4)$ \\
\hline $\mathrm{H} 13 \mathrm{~A}$ & -0.24650 & 0.86990 & 0.74910 & $0.0440^{*}$ & $0.686(4)$ \\
\hline
\end{tabular}




\begin{tabular}{llllll} 
H14A & -0.10610 & 0.72290 & 0.78230 & $0.0460 *$ & $0.314(4)$ \\
H15A & -0.04950 & 0.78040 & 0.86230 & $0.0460^{*}$ & $0.314(4)$ \\
H16A & 0.25340 & 0.82040 & 0.79120 & $0.0440^{*}$ & $0.314(4)$ \\
H17A & 0.28080 & 0.73790 & 0.81100 & $0.0440^{*}$ & $0.314(4)$ \\
H18A & -0.34390 & 0.81460 & 0.74010 & $0.0440^{*}$ & $0.314(4)$ \\
H19A & -0.15090 & 0.87100 & 0.76060 & $0.0440^{*}$ & $0.314(4)$ \\
\hline
\end{tabular}

Atomic displacement parameters $\left(\AA^{2}\right)$

\begin{tabular}{|c|c|c|c|c|c|c|}
\hline & $U^{11}$ & $U^{22}$ & $U^{33}$ & $U^{12}$ & $U^{13}$ & $U^{23}$ \\
\hline $\mathrm{O} 2 \mathrm{~B}$ & $0.0472(13)$ & 0.0563 (16) & $0.0297(14)$ & 0.0095 (12) & $0.0168(11)$ & $0.0083(12)$ \\
\hline O11B & $0.0356(9)$ & $0.0369(10)$ & $0.0579(12)$ & $0.0124(8)$ & $0.0087(8)$ & $0.0063(9)$ \\
\hline O12B & $0.0492(11)$ & $0.0640(13)$ & $0.0484(12)$ & $0.0080(10)$ & $0.0245(9)$ & $-0.0040(10)$ \\
\hline O31B & $0.0774(15)$ & $0.0847(18)$ & $0.0749(17)$ & $-0.0226(13)$ & $-0.0181(12)$ & $0.0525(14)$ \\
\hline O32B & $0.0708(15)$ & $0.0894(18)$ & $0.0629(15)$ & $0.0492(13)$ & $-0.0082(11)$ & $0.0045(12)$ \\
\hline O51B & $0.0542(11)$ & $0.0636(13)$ & $0.0680(14)$ & $0.0066(10)$ & $0.0346(10)$ & $-0.0115(11)$ \\
\hline O52B & $0.0918(15)$ & $0.0703(15)$ & $0.0456(13)$ & $0.0168(13)$ & $0.0323(11)$ & $0.0224(11)$ \\
\hline N3B & $0.0592(15)$ & $0.0338(13)$ & $0.0411(14)$ & 0.0003 (11) & $-0.0127(12)$ & $0.0001(11)$ \\
\hline N5B & $0.0464(12)$ & 0.0388 (13) & 0.0405 (13) & $-0.0040(10)$ & $0.0189(10)$ & $-0.0056(11)$ \\
\hline C1B & $0.0259(11)$ & $0.0216(12)$ & $0.0323(13)$ & $-0.0038(9)$ & $0.0021(9)$ & $-0.0035(10)$ \\
\hline $\mathrm{C} 2 \mathrm{~B}$ & $0.0341(12)$ & $0.0270(13)$ & $0.0280(13)$ & $-0.0057(10)$ & $0.0044(10)$ & $-0.0019(10)$ \\
\hline C3B & $0.0361(12)$ & $0.0241(12)$ & $0.0300(14)$ & $-0.0006(10)$ & $-0.0049(10)$ & $0.0015(10)$ \\
\hline C4B & $0.0256(11)$ & $0.0245(12)$ & $0.0406(15)$ & $-0.0010(10)$ & $-0.0006(10)$ & $-0.0054(11)$ \\
\hline C5B & $0.0297(11)$ & $0.0257(12)$ & $0.0285(13)$ & $-0.0056(10)$ & $0.0077(10)$ & $-0.0023(10)$ \\
\hline C6B & $0.0288(11)$ & $0.0214(12)$ & $0.0275(13)$ & -0.0017 (9) & $-0.0001(9)$ & $0.0021(10)$ \\
\hline $\mathrm{C} 11 \mathrm{~B}$ & $0.0292(12)$ & $0.0284(13)$ & $0.0466(16)$ & $-0.0028(10)$ & 0.0068 (11) & $-0.0055(12)$ \\
\hline $\mathrm{O} 21 \mathrm{~B}$ & $0.043(4)$ & $0.059(5)$ & $0.053(4)$ & $0.008(3)$ & $0.009(3)$ & $0.025(4)$ \\
\hline N1A & $0.0254(9)$ & $0.0319(11)$ & $0.0304(11)$ & $0.0014(8)$ & $0.0038(8)$ & $-0.0007(9)$ \\
\hline N8A & $0.0333(11)$ & $0.0476(13)$ & $0.0299(12)$ & $0.0157(10)$ & $0.0051(9)$ & $0.0034(10)$ \\
\hline $\mathrm{C} 2 \mathrm{~A}$ & $0.0254(11)$ & $0.0378(14)$ & $0.0427(15)$ & $0.0059(10)$ & $0.0006(10)$ & $-0.0026(12)$ \\
\hline $\mathrm{C} 3 \mathrm{~A}$ & $0.0358(13)$ & 0.0339 (14) & $0.0464(16)$ & $0.0066(11)$ & $-0.0044(11)$ & $0.0003(12)$ \\
\hline $\mathrm{C} 4 \mathrm{~A}$ & $0.0442(14)$ & $0.0384(15)$ & $0.0365(15)$ & $-0.0024(12)$ & $-0.0061(11)$ & $0.0074(12)$ \\
\hline C5A & $0.0370(13)$ & 0.0508 (17) & $0.0308(14)$ & $-0.0005(12)$ & $0.0075(10)$ & $0.0080(12)$ \\
\hline C6A & $0.0340(12)$ & 0.0413 (15) & 0.0262 (13) & $0.0081(11)$ & $0.0029(10)$ & $-0.0052(11)$ \\
\hline C7A & $0.0270(11)$ & $0.0226(12)$ & $0.0291(13)$ & $-0.0006(9)$ & $0.0014(9)$ & $-0.0035(10)$ \\
\hline C9A & $0.042(3)$ & $0.037(4)$ & $0.0292(18)$ & $0.001(2)$ & $0.000(2)$ & $0.005(3)$ \\
\hline C10A & $0.047(2)$ & $0.041(2)$ & 0.0263 (17) & $-0.0052(17)$ & $0.0070(16)$ & -0.0033 (19) \\
\hline C11A & $0.0363(13)$ & $0.0419(15)$ & $0.0335(14)$ & $-0.0014(11)$ & $0.0150(11)$ & $-0.0058(12)$ \\
\hline $\mathrm{C} 13 \mathrm{~A}$ & $0.042(3)$ & $0.037(4)$ & $0.0292(18)$ & $0.001(2)$ & $0.000(2)$ & $0.005(3)$ \\
\hline $\mathrm{C} 12 \mathrm{~A}$ & $0.047(2)$ & $0.041(2)$ & $0.0263(17)$ & $-0.0052(17)$ & $0.0070(16)$ & $-0.0033(19)$ \\
\hline
\end{tabular}

Geometric parameters $(\AA, \stackrel{\circ}{)})$

\begin{tabular}{llll}
\hline $\mathrm{O} 2 \mathrm{~B}-\mathrm{C} 2 \mathrm{~B}$ & $1.281(3)$ & $\mathrm{C} 3 \mathrm{~A}-\mathrm{C} 4 \mathrm{~A}$ & $1.522(4)$ \\
$\mathrm{O} 11 \mathrm{~B}-\mathrm{C} 11 \mathrm{~B}$ & $1.247(3)$ & $\mathrm{C} 4 \mathrm{~A}-\mathrm{C} 5 \mathrm{~A}$ & $1.520(4)$ \\
$\mathrm{O} 12 \mathrm{~B}-\mathrm{C} 11 \mathrm{~B}$ & $1.271(3)$ & $\mathrm{C} 5 \mathrm{~A}-\mathrm{C} 6 \mathrm{~A}$ & $1.531(4)$ \\
$\mathrm{O} 21 \mathrm{~B}-\mathrm{C} 6 \mathrm{~B}$ & $1.305(7)$ & $\mathrm{C} 6 \mathrm{~A}-\mathrm{C} 7 \mathrm{~A}$ & $1.493(3)$ \\
$\mathrm{O} 31 \mathrm{~B}-\mathrm{N} 3 \mathrm{~B}$ & $1.208(3)$ & $\mathrm{C} 9 \mathrm{~A}-\mathrm{C} 10 \mathrm{~A}$ & $1.510(6)$
\end{tabular}




\begin{tabular}{|c|c|c|c|}
\hline $\mathrm{O} 32 \mathrm{~B}-\mathrm{N} 3 \mathrm{~B}$ & $1.230(4)$ & $\mathrm{C} 10 \mathrm{~A}-\mathrm{C} 11 \mathrm{~A}$ & $1.503(5)$ \\
\hline $\mathrm{O} 51 \mathrm{~B}-\mathrm{N} 5 \mathrm{~B}$ & $1.217(3)$ & $\mathrm{C} 12 \mathrm{~A}-\mathrm{C} 13 \mathrm{~A}$ & $1.523(15)$ \\
\hline $\mathrm{O} 52 \mathrm{~B}-\mathrm{N} 5 \mathrm{~B}$ & $1.230(3)$ & $\mathrm{C} 2 \mathrm{~A}-\mathrm{H} 21 \mathrm{~A}$ & 0.9900 \\
\hline $\mathrm{O} 2 \mathrm{~B}-\mathrm{H} 2 \mathrm{~B}$ & 0.8400 & $\mathrm{C} 2 \mathrm{~A}-\mathrm{H} 22 \mathrm{~A}$ & 0.9900 \\
\hline $\mathrm{O} 21 \mathrm{~B}-\mathrm{H} 21 \mathrm{~B}$ & 0.8400 & $\mathrm{C} 3 \mathrm{~A}-\mathrm{H} 31 \mathrm{~A}$ & 0.9900 \\
\hline $\mathrm{N} 3 \mathrm{~B}-\mathrm{C} 3 \mathrm{~B}$ & $1.460(3)$ & $\mathrm{C} 3 \mathrm{~A}-\mathrm{H} 32 \mathrm{~A}$ & 0.9900 \\
\hline $\mathrm{N} 5 \mathrm{~B}-\mathrm{C} 5 \mathrm{~B}$ & $1.455(3)$ & $\mathrm{C} 4 \mathrm{~A}-\mathrm{H} 41 \mathrm{~A}$ & 0.9900 \\
\hline $\mathrm{N} 1 \mathrm{~A}-\mathrm{C} 2 \mathrm{~A}$ & $1.473(3)$ & $\mathrm{C} 4 \mathrm{~A}-\mathrm{H} 42 \mathrm{~A}$ & 0.9900 \\
\hline N1A-C11A & $1.473(3)$ & $\mathrm{C} 5 \mathrm{~A}-\mathrm{H} 51 \mathrm{~A}$ & 0.9900 \\
\hline $\mathrm{N} 1 \mathrm{~A}-\mathrm{C} 7 \mathrm{~A}$ & $1.314(3)$ & $\mathrm{C} 5 \mathrm{~A}-\mathrm{H} 52 \mathrm{~A}$ & 0.9900 \\
\hline $\mathrm{N} 8 \mathrm{~A}-\mathrm{C} 9 \mathrm{~A}$ & $1.467(6)$ & C6A-H61A & 0.9900 \\
\hline $\mathrm{N} 8 \mathrm{~A}-\mathrm{C} 13 \mathrm{~A}$ & $1.498(16)$ & $\mathrm{C} 6 \mathrm{~A}-\mathrm{H} 62 \mathrm{~A}$ & 0.9900 \\
\hline $\mathrm{N} 8 \mathrm{~A}-\mathrm{C} 7 \mathrm{~A}$ & $1.308(3)$ & C9A-H91A & 0.9900 \\
\hline N8A-H8A & $0.88(2)$ & $\mathrm{C} 9 \mathrm{~A}-\mathrm{H} 92 \mathrm{~A}$ & 0.9900 \\
\hline $\mathrm{C} 1 \mathrm{~B}-\mathrm{C} 11 \mathrm{~B}$ & $1.499(3)$ & $\mathrm{C} 10 \mathrm{~A}-\mathrm{H} 10 \mathrm{~A}$ & 0.9900 \\
\hline $\mathrm{C} 1 \mathrm{~B}-\mathrm{C} 6 \mathrm{~B}$ & $1.387(3)$ & $\mathrm{C} 10 \mathrm{~A}-\mathrm{H} 11 \mathrm{~A}$ & 0.9900 \\
\hline $\mathrm{C} 1 \mathrm{~B}-\mathrm{C} 2 \mathrm{~B}$ & $1.406(3)$ & $\mathrm{C} 11 \mathrm{~A}-\mathrm{H} 12 \mathrm{~A}$ & 0.9900 \\
\hline $\mathrm{C} 2 \mathrm{~B}-\mathrm{C} 3 \mathrm{~B}$ & $1.406(3)$ & $\mathrm{C} 11 \mathrm{~A}-\mathrm{H} 13 \mathrm{~A}$ & 0.9900 \\
\hline $\mathrm{C} 3 \mathrm{~B}-\mathrm{C} 4 \mathrm{~B}$ & $1.372(3)$ & $\mathrm{C} 12 \mathrm{~A}-\mathrm{H} 14 \mathrm{~A}$ & 0.9900 \\
\hline $\mathrm{C} 4 \mathrm{~B}-\mathrm{C} 5 \mathrm{~B}$ & $1.376(3)$ & $\mathrm{C} 12 \mathrm{~A}-\mathrm{H} 15 \mathrm{~A}$ & 0.9900 \\
\hline $\mathrm{C} 5 \mathrm{~B}-\mathrm{C} 6 \mathrm{~B}$ & $1.391(3)$ & $\mathrm{C} 13 \mathrm{~A}-\mathrm{H} 16 \mathrm{~A}$ & 0.9900 \\
\hline $\mathrm{C} 2 \mathrm{~B}-\mathrm{H} 61 \mathrm{~B}$ & 0.9500 & $\mathrm{C} 13 \mathrm{~A}-\mathrm{H} 17 \mathrm{~A}$ & 0.9900 \\
\hline $\mathrm{C} 4 \mathrm{~B}-\mathrm{H} 4 \mathrm{~B}$ & 0.9500 & $\mathrm{C} 11 \mathrm{~A}-\mathrm{H} 18 \mathrm{~A}$ & 0.9900 \\
\hline C6B-H6B & 0.9500 & $\mathrm{C} 11 \mathrm{~A}-\mathrm{H} 19 \mathrm{~A}$ & 0.9900 \\
\hline $\mathrm{C} 2 \mathrm{~A}-\mathrm{C} 3 \mathrm{~A}$ & $1.511(3)$ & & \\
\hline $\mathrm{C} 2 \mathrm{~B}-\mathrm{O} 2 \mathrm{~B}-\mathrm{H} 2 \mathrm{~B}$ & 109.00 & $\mathrm{C} 9 \mathrm{~A}-\mathrm{C} 10 \mathrm{~A}-\mathrm{C} 11 \mathrm{~A}$ & $110.2(3)$ \\
\hline $\mathrm{C} 6 \mathrm{~B}-\mathrm{O} 21 \mathrm{~B}-\mathrm{H} 21 \mathrm{~B}$ & 110.00 & $\mathrm{~N} 1 \mathrm{~A}-\mathrm{C} 11 \mathrm{~A}-\mathrm{C} 10 \mathrm{~A}$ & $111.3(2)$ \\
\hline $\mathrm{O} 32 \mathrm{~B}-\mathrm{N} 3 \mathrm{~B}-\mathrm{C} 3 \mathrm{~B}$ & $117.7(2)$ & $\mathrm{N} 8 \mathrm{~A}-\mathrm{C} 13 \mathrm{~A}-\mathrm{C} 12 \mathrm{~A}$ & $104.7(9)$ \\
\hline $\mathrm{O} 31 \mathrm{~B}-\mathrm{N} 3 \mathrm{~B}-\mathrm{O} 32 \mathrm{~B}$ & $123.7(2)$ & $\mathrm{N} 1 \mathrm{~A}-\mathrm{C} 2 \mathrm{~A}-\mathrm{H} 21 \mathrm{~A}$ & 109.00 \\
\hline $\mathrm{O} 31 \mathrm{~B}-\mathrm{N} 3 \mathrm{~B}-\mathrm{C} 3 \mathrm{~B}$ & $118.6(2)$ & $\mathrm{N} 1 \mathrm{~A}-\mathrm{C} 2 \mathrm{~A}-\mathrm{H} 22 \mathrm{~A}$ & 109.00 \\
\hline $\mathrm{O} 52 \mathrm{~B}-\mathrm{N} 5 \mathrm{~B}-\mathrm{C} 5 \mathrm{~B}$ & $117.7(2)$ & $\mathrm{C} 3 \mathrm{~A}-\mathrm{C} 2 \mathrm{~A}-\mathrm{H} 21 \mathrm{~A}$ & 109.00 \\
\hline $\mathrm{O} 51 \mathrm{~B}-\mathrm{N} 5 \mathrm{~B}-\mathrm{C} 5 \mathrm{~B}$ & $118.7(2)$ & $\mathrm{C} 3 \mathrm{~A}-\mathrm{C} 2 \mathrm{~A}-\mathrm{H} 22 \mathrm{~A}$ & 109.00 \\
\hline $\mathrm{O} 51 \mathrm{~B}-\mathrm{N} 5 \mathrm{~B}-\mathrm{O} 52 \mathrm{~B}$ & $123.5(2)$ & $\mathrm{H} 21 \mathrm{~A}-\mathrm{C} 2 \mathrm{~A}-\mathrm{H} 22 \mathrm{~A}$ & 108.00 \\
\hline $\mathrm{C} 2 \mathrm{~A}-\mathrm{N} 1 \mathrm{~A}-\mathrm{C} 11 \mathrm{~A}$ & $116.36(18)$ & $\mathrm{C} 2 \mathrm{~A}-\mathrm{C} 3 \mathrm{~A}-\mathrm{H} 31 \mathrm{~A}$ & 109.00 \\
\hline $\mathrm{C} 7 \mathrm{~A}-\mathrm{N} 1 \mathrm{~A}-\mathrm{C} 11 \mathrm{~A}$ & $121.90(19)$ & $\mathrm{C} 2 \mathrm{~A}-\mathrm{C} 3 \mathrm{~A}-\mathrm{H} 32 \mathrm{~A}$ & 109.00 \\
\hline $\mathrm{C} 2 \mathrm{~A}-\mathrm{N} 1 \mathrm{~A}-\mathrm{C} 7 \mathrm{~A}$ & $121.74(19)$ & $\mathrm{C} 4 \mathrm{~A}-\mathrm{C} 3 \mathrm{~A}-\mathrm{H} 31 \mathrm{~A}$ & 109.00 \\
\hline $\mathrm{C} 7 \mathrm{~A}-\mathrm{N} 8 \mathrm{~A}-\mathrm{C} 13 \mathrm{~A}$ & $122.0(5)$ & $\mathrm{C} 4 \mathrm{~A}-\mathrm{C} 3 \mathrm{~A}-\mathrm{H} 32 \mathrm{~A}$ & 109.00 \\
\hline $\mathrm{C} 7 \mathrm{~A}-\mathrm{N} 8 \mathrm{~A}-\mathrm{C} 9 \mathrm{~A}$ & $122.5(3)$ & $\mathrm{H} 31 \mathrm{~A}-\mathrm{C} 3 \mathrm{~A}-\mathrm{H} 32 \mathrm{~A}$ & 108.00 \\
\hline $\mathrm{C} 9 \mathrm{~A}-\mathrm{N} 8 \mathrm{~A}-\mathrm{H} 8 \mathrm{~A}$ & $119.3(14)$ & $\mathrm{C} 3 \mathrm{~A}-\mathrm{C} 4 \mathrm{~A}-\mathrm{H} 41 \mathrm{~A}$ & 109.00 \\
\hline $\mathrm{C} 13 \mathrm{~A}-\mathrm{N} 8 \mathrm{~A}-\mathrm{H} 8 \mathrm{~A}$ & $119.6(15)$ & $\mathrm{C} 3 \mathrm{~A}-\mathrm{C} 4 \mathrm{~A}-\mathrm{H} 42 \mathrm{~A}$ & 109.00 \\
\hline $\mathrm{C} 7 \mathrm{~A}-\mathrm{N} 8 \mathrm{~A}-\mathrm{H} 8 \mathrm{~A}$ & $117.0(14)$ & $\mathrm{C} 5 \mathrm{~A}-\mathrm{C} 4 \mathrm{~A}-\mathrm{H} 41 \mathrm{~A}$ & 109.00 \\
\hline $\mathrm{C} 2 \mathrm{~B}-\mathrm{C} 1 \mathrm{~B}-\mathrm{C} 6 \mathrm{~B}$ & $120.78(18)$ & $\mathrm{C} 5 \mathrm{~A}-\mathrm{C} 4 \mathrm{~A}-\mathrm{H} 42 \mathrm{~A}$ & 109.00 \\
\hline $\mathrm{C} 2 \mathrm{~B}-\mathrm{C} 1 \mathrm{~B}-\mathrm{C} 11 \mathrm{~B}$ & $119.6(2)$ & $\mathrm{H} 41 \mathrm{~A}-\mathrm{C} 4 \mathrm{~A}-\mathrm{H} 42 \mathrm{~A}$ & 108.00 \\
\hline $\mathrm{C} 6 \mathrm{~B}-\mathrm{C} 1 \mathrm{~B}-\mathrm{C} 11 \mathrm{~B}$ & $119.6(2)$ & $\mathrm{C} 4 \mathrm{~A}-\mathrm{C} 5 \mathrm{~A}-\mathrm{H} 51 \mathrm{~A}$ & 109.00 \\
\hline $\mathrm{O} 2 \mathrm{~B}-\mathrm{C} 2 \mathrm{~B}-\mathrm{C} 1 \mathrm{~B}$ & $122.0(2)$ & $\mathrm{C} 4 \mathrm{~A}-\mathrm{C} 5 \mathrm{~A}-\mathrm{H} 52 \mathrm{~A}$ & 109.00 \\
\hline $\mathrm{C} 1 \mathrm{~B}-\mathrm{C} 2 \mathrm{~B}-\mathrm{C} 3 \mathrm{~B}$ & $117.4(2)$ & $\mathrm{C} 6 \mathrm{~A}-\mathrm{C} 5 \mathrm{~A}-\mathrm{H} 51 \mathrm{~A}$ & 109.00 \\
\hline $\mathrm{O} 2 \mathrm{~B}-\mathrm{C} 2 \mathrm{~B}-\mathrm{C} 3 \mathrm{~B}$ & $120.5(2)$ & $\mathrm{C} 6 \mathrm{~A}-\mathrm{C} 5 \mathrm{~A}-\mathrm{H} 52 \mathrm{~A}$ & 109.00 \\
\hline
\end{tabular}




\begin{tabular}{|c|c|}
\hline $\mathrm{N} 3 \mathrm{~B}-\mathrm{C} 3 \mathrm{~B}-\mathrm{C} 4 \mathrm{~B}$ & $117.1(2)$ \\
\hline $\mathrm{C} 2 \mathrm{~B}-\mathrm{C} 3 \mathrm{~B}-\mathrm{C} 4 \mathrm{~B}$ & $122.3(2)$ \\
\hline $\mathrm{N} 3 \mathrm{~B}-\mathrm{C} 3 \mathrm{~B}-\mathrm{C} 2 \mathrm{~B}$ & $120.6(2)$ \\
\hline $\mathrm{C} 3 \mathrm{~B}-\mathrm{C} 4 \mathrm{~B}-\mathrm{C} 5 \mathrm{~B}$ & $118.84(19)$ \\
\hline $\mathrm{C} 4 \mathrm{~B}-\mathrm{C} 5 \mathrm{~B}-\mathrm{C} 6 \mathrm{~B}$ & $121.43(19)$ \\
\hline $\mathrm{N} 5 \mathrm{~B}-\mathrm{C} 5 \mathrm{~B}-\mathrm{C} 4 \mathrm{~B}$ & $118.69(18)$ \\
\hline $\mathrm{N} 5 \mathrm{~B}-\mathrm{C} 5 \mathrm{~B}-\mathrm{C} 6 \mathrm{~B}$ & $119.88(19)$ \\
\hline $\mathrm{O} 21 \mathrm{~B}-\mathrm{C} 6 \mathrm{~B}-\mathrm{C} 1 \mathrm{~B}$ & $118.7(3)$ \\
\hline $\mathrm{O} 21 \mathrm{~B}-\mathrm{C} 6 \mathrm{~B}-\mathrm{C} 5 \mathrm{~B}$ & $122.0(3)$ \\
\hline $\mathrm{C} 1 \mathrm{~B}-\mathrm{C} 6 \mathrm{~B}-\mathrm{C} 5 \mathrm{~B}$ & $119.23(19)$ \\
\hline $\mathrm{O} 12 \mathrm{~B}-\mathrm{C} 11 \mathrm{~B}-\mathrm{C} 1 \mathrm{~B}$ & $116.6(2)$ \\
\hline $\mathrm{O} 11 \mathrm{~B}-\mathrm{C} 11 \mathrm{~B}-\mathrm{C} 1 \mathrm{~B}$ & $119.3(2)$ \\
\hline $\mathrm{O} 11 \mathrm{~B}-\mathrm{C} 11 \mathrm{~B}-\mathrm{O} 12 \mathrm{~B}$ & $124.1(2)$ \\
\hline $\mathrm{C} 3 \mathrm{~B}-\mathrm{C} 2 \mathrm{~B}-\mathrm{H} 61 \mathrm{~B}$ & 121.00 \\
\hline $\mathrm{C} 1 \mathrm{~B}-\mathrm{C} 2 \mathrm{~B}-\mathrm{H} 61 \mathrm{~B}$ & 122.00 \\
\hline $\mathrm{C} 5 \mathrm{~B}-\mathrm{C} 4 \mathrm{~B}-\mathrm{H} 4 \mathrm{~B}$ & 121.00 \\
\hline $\mathrm{C} 3 \mathrm{~B}-\mathrm{C} 4 \mathrm{~B}-\mathrm{H} 4 \mathrm{~B}$ & 121.00 \\
\hline $\mathrm{C} 1 \mathrm{~B}-\mathrm{C} 6 \mathrm{~B}-\mathrm{H} 6 \mathrm{~B}$ & 120.00 \\
\hline $\mathrm{C} 5 \mathrm{~B}-\mathrm{C} 6 \mathrm{~B}-\mathrm{H} 6 \mathrm{~B}$ & 121.00 \\
\hline $\mathrm{N} 1 \mathrm{~A}-\mathrm{C} 2 \mathrm{~A}-\mathrm{C} 3 \mathrm{~A}$ & $114.04(18)$ \\
\hline $\mathrm{C} 2 \mathrm{~A}-\mathrm{C} 3 \mathrm{~A}-\mathrm{C} 4 \mathrm{~A}$ & $114.2(2)$ \\
\hline $\mathrm{C} 3 \mathrm{~A}-\mathrm{C} 4 \mathrm{~A}-\mathrm{C} 5 \mathrm{~A}$ & $114.5(2)$ \\
\hline $\mathrm{C} 4 \mathrm{~A}-\mathrm{C} 5 \mathrm{~A}-\mathrm{C} 6 \mathrm{~A}$ & $114.4(2)$ \\
\hline $\mathrm{C} 5 \mathrm{~A}-\mathrm{C} 6 \mathrm{~A}-\mathrm{C} 7 \mathrm{~A}$ & $112.9(2)$ \\
\hline $\mathrm{N} 1 \mathrm{~A}-\mathrm{C} 7 \mathrm{~A}-\mathrm{N} 8 \mathrm{~A}$ & $121.8(2)$ \\
\hline $\mathrm{N} 1 \mathrm{~A}-\mathrm{C} 7 \mathrm{~A}-\mathrm{C} 6 \mathrm{~A}$ & $120.35(19)$ \\
\hline $\mathrm{N} 8 \mathrm{~A}-\mathrm{C} 7 \mathrm{~A}-\mathrm{C} 6 \mathrm{~A}$ & $117.82(19)$ \\
\hline $\mathrm{N} 8 \mathrm{~A}-\mathrm{C} 9 \mathrm{~A}-\mathrm{C} 10 \mathrm{~A}$ & $106.7(3)$ \\
\hline $\mathrm{O} 31 \mathrm{~B}-\mathrm{N} 3 \mathrm{~B}-\mathrm{C} 3 \mathrm{~B}-\mathrm{C} 2 \mathrm{~B}$ & $23.9(3)$ \\
\hline $\mathrm{O} 31 \mathrm{~B}-\mathrm{N} 3 \mathrm{~B}-\mathrm{C} 3 \mathrm{~B}-\mathrm{C} 4 \mathrm{~B}$ & $-157.2(2)$ \\
\hline $\mathrm{O} 32 \mathrm{~B}-\mathrm{N} 3 \mathrm{~B}-\mathrm{C} 3 \mathrm{~B}-\mathrm{C} 2 \mathrm{~B}$ & $-155.2(2)$ \\
\hline $\mathrm{O} 32 \mathrm{~B}-\mathrm{N} 3 \mathrm{~B}-\mathrm{C} 3 \mathrm{~B}-\mathrm{C} 4 \mathrm{~B}$ & $23.8(3)$ \\
\hline $\mathrm{O} 51 \mathrm{~B}-\mathrm{N} 5 \mathrm{~B}-\mathrm{C} 5 \mathrm{~B}-\mathrm{C} 4 \mathrm{~B}$ & $3.7(3)$ \\
\hline $\mathrm{O} 51 \mathrm{~B}-\mathrm{N} 5 \mathrm{~B}-\mathrm{C} 5 \mathrm{~B}-\mathrm{C} 6 \mathrm{~B}$ & $-176.8(2)$ \\
\hline $\mathrm{O} 52 \mathrm{~B}-\mathrm{N} 5 \mathrm{~B}-\mathrm{C} 5 \mathrm{~B}-\mathrm{C} 4 \mathrm{~B}$ & $-177.5(2)$ \\
\hline $\mathrm{O} 52 \mathrm{~B}-\mathrm{N} 5 \mathrm{~B}-\mathrm{C} 5 \mathrm{~B}-\mathrm{C} 6 \mathrm{~B}$ & $2.0(3)$ \\
\hline $\mathrm{C} 2 \mathrm{~A}-\mathrm{N} 1 \mathrm{~A}-\mathrm{C} 7 \mathrm{~A}-\mathrm{N} 8 \mathrm{~A}$ & $-176.4(2)$ \\
\hline $\mathrm{C} 2 \mathrm{~A}-\mathrm{N} 1 \mathrm{~A}-\mathrm{C} 7 \mathrm{~A}-\mathrm{C} 6 \mathrm{~A}$ & $6.0(3)$ \\
\hline $\mathrm{C} 11 \mathrm{~A}-\mathrm{N} 1 \mathrm{~A}-\mathrm{C} 7 \mathrm{~A}-\mathrm{N} 8 \mathrm{~A}$ & $2.7(3)$ \\
\hline $\mathrm{C} 11 \mathrm{~A}-\mathrm{N} 1 \mathrm{~A}-\mathrm{C} 7 \mathrm{~A}-\mathrm{C} 6 \mathrm{~A}$ & $-175.0(2)$ \\
\hline $\mathrm{C} 2 \mathrm{~A}-\mathrm{N} 1 \mathrm{~A}-\mathrm{C} 11 \mathrm{~A}-\mathrm{C} 10 \mathrm{~A}$ & $-163.0(2)$ \\
\hline $\mathrm{C} 7 \mathrm{~A}-\mathrm{N} 1 \mathrm{~A}-\mathrm{C} 2 \mathrm{~A}-\mathrm{C} 3 \mathrm{~A}$ & $-71.7(3)$ \\
\hline $\mathrm{C} 11 \mathrm{~A}-\mathrm{N} 1 \mathrm{~A}-\mathrm{C} 2 \mathrm{~A}-\mathrm{C} 3 \mathrm{~A}$ & $109.2(2)$ \\
\hline $\mathrm{C} 7 \mathrm{~A}-\mathrm{N} 1 \mathrm{~A}-\mathrm{C} 11 \mathrm{~A}-\mathrm{C} 10 \mathrm{~A}$ & $17.9(3)$ \\
\hline $\mathrm{C} 7 \mathrm{~A}-\mathrm{N} 8 \mathrm{~A}-\mathrm{C} 9 \mathrm{~A}-\mathrm{C} 10 \mathrm{~A}$ & $-38.8(4)$ \\
\hline $\mathrm{C} 9 \mathrm{~A}-\mathrm{N} 8 \mathrm{~A}-\mathrm{C} 7 \mathrm{~A}-\mathrm{C} 6 \mathrm{~A}$ & $-173.2(3)$ \\
\hline $\mathrm{C} 9 \mathrm{~A}-\mathrm{N} 8 \mathrm{~A}-\mathrm{C} 7 \mathrm{~A}-\mathrm{N} 1 \mathrm{~A}$ & $9.1(4)$ \\
\hline
\end{tabular}

H51A-C5A-H52A

C5A-C6A-H61A $\quad 109.00$

$\mathrm{C} 5 \mathrm{~A}-\mathrm{C} 6 \mathrm{~A}-\mathrm{H} 62 \mathrm{~A} \quad 109.00$

C7A-C6A-H61A $\quad 109.00$

C7A-C6A-H62A $\quad 109.00$

H61A-C6A-H62A $\quad 108.00$

$\mathrm{N} 8 \mathrm{~A}-\mathrm{C} 9 \mathrm{~A}-\mathrm{H} 91 \mathrm{~A} \quad 110.00$

$\mathrm{N} 8 \mathrm{~A}-\mathrm{C} 9 \mathrm{~A}-\mathrm{H} 92 \mathrm{~A} \quad 110.00$

C10A-C9A-H91A $\quad 110.00$

C10A-C9A-H92A $\quad 110.00$

H91A-C9A-H92A $\quad 109.00$

C9A $-\mathrm{C} 10 \mathrm{~A}-\mathrm{H} 10 \mathrm{~A} \quad 110.00$

C9A $-\mathrm{C} 10 \mathrm{~A}-\mathrm{H} 11 \mathrm{~A} \quad 110.00$

C11A-C10A-H10A $\quad 110.00$

$\mathrm{C} 11 \mathrm{~A}-\mathrm{C} 10 \mathrm{~A}-\mathrm{H} 11 \mathrm{~A} \quad 110.00$

$\mathrm{H} 10 \mathrm{~A}-\mathrm{C} 10 \mathrm{~A}-\mathrm{H} 11 \mathrm{~A} \quad 108.00$

$\mathrm{N} 1 \mathrm{~A}-\mathrm{C} 11 \mathrm{~A}-\mathrm{H} 12 \mathrm{~A} \quad 109.00$

$\mathrm{N} 1 \mathrm{~A}-\mathrm{C} 11 \mathrm{~A}-\mathrm{H} 13 \mathrm{~A} \quad 109.00$

C10A-C11A-H12A $\quad 109.00$

C10A-C11A-H13A $\quad 109.00$

H12A-C11A-H13A $\quad 108.00$

$\mathrm{C} 13 \mathrm{~A}-\mathrm{C} 12 \mathrm{~A}-\mathrm{H} 15 \mathrm{~A} \quad 110.00$

H14A-C12A-H15A $\quad 108.00$

$\mathrm{N} 8 \mathrm{~A}-\mathrm{C} 13 \mathrm{~A}-\mathrm{H} 16 \mathrm{~A} \quad 111.00$

$\mathrm{N} 8 \mathrm{~A}-\mathrm{C} 13 \mathrm{~A}-\mathrm{H} 17 \mathrm{~A} \quad 111.00$

$\mathrm{C} 12 \mathrm{~A}-\mathrm{C} 13 \mathrm{~A}-\mathrm{H} 16 \mathrm{~A} \quad 111.00$

$\mathrm{C} 12 \mathrm{~A}-\mathrm{C} 13 \mathrm{~A}-\mathrm{H} 17 \mathrm{~A} \quad 111.00$

H16A-C13A-H17A $\quad 109.00$

$\mathrm{C} 6 \mathrm{~B}-\mathrm{C} 1 \mathrm{~B}-\mathrm{C} 11 \mathrm{~B}-\mathrm{O} 11 \mathrm{~B}$

$\mathrm{C} 6 \mathrm{~B}-\mathrm{C} 1 \mathrm{~B}-\mathrm{C} 11 \mathrm{~B}-\mathrm{O} 12 \mathrm{~B} \quad-175.8(2)$

$\mathrm{C} 2 \mathrm{~B}-\mathrm{C} 1 \mathrm{~B}-\mathrm{C} 11 \mathrm{~B}-\mathrm{O} 11 \mathrm{~B} \quad-179.4(2)$

$\mathrm{C} 2 \mathrm{~B}-\mathrm{C} 1 \mathrm{~B}-\mathrm{C} 11 \mathrm{~B}-\mathrm{O} 12 \mathrm{~B} \quad 1.8(3)$

$\mathrm{C} 11 \mathrm{~B}-\mathrm{C} 1 \mathrm{~B}-\mathrm{C} 6 \mathrm{~B}-\mathrm{C} 5 \mathrm{~B} \quad 175.2(2)$

$\mathrm{O} 2 \mathrm{~B}-\mathrm{C} 2 \mathrm{~B}-\mathrm{C} 3 \mathrm{~B}-\mathrm{N} 3 \mathrm{~B} \quad 5.6(4)$

$\mathrm{O} 2 \mathrm{~B}-\mathrm{C} 2 \mathrm{~B}-\mathrm{C} 3 \mathrm{~B}-\mathrm{C} 4 \mathrm{~B} \quad-173.3$ (2)

$\mathrm{C} 1 \mathrm{~B}-\mathrm{C} 2 \mathrm{~B}-\mathrm{C} 3 \mathrm{~B}-\mathrm{N} 3 \mathrm{~B} \quad-178.5(2)$

$\mathrm{C} 1 \mathrm{~B}-\mathrm{C} 2 \mathrm{~B}-\mathrm{C} 3 \mathrm{~B}-\mathrm{C} 4 \mathrm{~B} \quad 2.7(3)$

$\mathrm{C} 2 \mathrm{~B}-\mathrm{C} 3 \mathrm{~B}-\mathrm{C} 4 \mathrm{~B}-\mathrm{C} 5 \mathrm{~B} \quad-2.9(3)$

$\mathrm{N} 3 \mathrm{~B}-\mathrm{C} 3 \mathrm{~B}-\mathrm{C} 4 \mathrm{~B}-\mathrm{C} 5 \mathrm{~B} \quad 178.3$ (2)

$\mathrm{C} 3 \mathrm{~B}-\mathrm{C} 4 \mathrm{~B}-\mathrm{C} 5 \mathrm{~B}-\mathrm{C} 6 \mathrm{~B} \quad 0.4(3)$

$\mathrm{C} 3 \mathrm{~B}-\mathrm{C} 4 \mathrm{~B}-\mathrm{C} 5 \mathrm{~B}-\mathrm{N} 5 \mathrm{~B} \quad 179.9$ (2)

$\mathrm{N} 5 \mathrm{~B}-\mathrm{C} 5 \mathrm{~B}-\mathrm{C} 6 \mathrm{~B}-\mathrm{C} 1 \mathrm{~B} \quad-177.36$ (19)

$\mathrm{C} 4 \mathrm{~B}-\mathrm{C} 5 \mathrm{~B}-\mathrm{C} 6 \mathrm{~B}-\mathrm{C} 1 \mathrm{~B} \quad 2.1(3)$

$\mathrm{N} 1 \mathrm{~A}-\mathrm{C} 2 \mathrm{~A}-\mathrm{C} 3 \mathrm{~A}-\mathrm{C} 4 \mathrm{~A} \quad 78.8(3)$

$\mathrm{C} 2 \mathrm{~A}-\mathrm{C} 3 \mathrm{~A}-\mathrm{C} 4 \mathrm{~A}-\mathrm{C} 5 \mathrm{~A} \quad-57.5(3)$

$\mathrm{C} 3 \mathrm{~A}-\mathrm{C} 4 \mathrm{~A}-\mathrm{C} 5 \mathrm{~A}-\mathrm{C} 6 \mathrm{~A} \quad 61.0(3)$

$\mathrm{C} 4 \mathrm{~A}-\mathrm{C} 5 \mathrm{~A}-\mathrm{C} 6 \mathrm{~A}-\mathrm{C} 7 \mathrm{~A} \quad-82.0(3)$ 


$\begin{array}{llll}\mathrm{C} 6 \mathrm{~B}-\mathrm{C} 1 \mathrm{~B}-\mathrm{C} 2 \mathrm{~B}-\mathrm{C} 3 \mathrm{~B} & 0.0(3) & \mathrm{C} 5 \mathrm{~A}-\mathrm{C} 6 \mathrm{~A}-\mathrm{C} 7 \mathrm{~A}-\mathrm{N} 1 \mathrm{~A} & 63.3(3) \\ \mathrm{C} 11 \mathrm{~B}-\mathrm{C} 1 \mathrm{~B}-\mathrm{C} 2 \mathrm{~B}-\mathrm{O} 2 \mathrm{~B} & -1.6(3) & \mathrm{C} 5 \mathrm{~A}-\mathrm{C} 6 \mathrm{~A}-\mathrm{C} 7 \mathrm{~A}-\mathrm{N} 8 \mathrm{~A} & -114.5(2) \\ \mathrm{C} 11 \mathrm{~B}-\mathrm{C} 1 \mathrm{~B}-\mathrm{C} 2 \mathrm{~B}-\mathrm{C} 3 \mathrm{~B} & -177.5(2) & \mathrm{N} 8 \mathrm{~A}-\mathrm{C} 9 \mathrm{~A}-\mathrm{C} 10 \mathrm{~A}-\mathrm{C} 11 \mathrm{~A} & 56.2(4) \\ \mathrm{C} 2 \mathrm{~B}-\mathrm{C} 1 \mathrm{~B}-\mathrm{C} 6 \mathrm{~B}-\mathrm{C} 5 \mathrm{~B} & -2.3(3) & \mathrm{C} 9 \mathrm{~A}-\mathrm{C} 10 \mathrm{~A}-\mathrm{C} 11 \mathrm{~A}-\mathrm{N} 1 \mathrm{~A} & -47.7(4) \\ \mathrm{C} 6 \mathrm{~B}-\mathrm{C} 1 \mathrm{~B}-\mathrm{C} 2 \mathrm{~B}-\mathrm{O} 2 \mathrm{~B} & 175.9(2) & & \end{array}$

Hydrogen-bond geometry $\left(\AA,{ }^{\circ}\right)$

\begin{tabular}{lllll}
\hline$D-\mathrm{H} \cdots A$ & $D-\mathrm{H}$ & $\mathrm{H} \cdots A$ & $D \cdots A$ & $D-\mathrm{H} \cdots A$ \\
\hline $\mathrm{N} 8 A-\mathrm{H} 8 A \cdots \mathrm{O} 11 B$ & $0.88(2)$ & $1.99(2)$ & $2.871(3)$ & $176(2)$ \\
$\mathrm{O} 2 B-\mathrm{H} 2 B \cdots \mathrm{O} 12 B$ & 0.84 & 1.72 & $2.473(3)$ & 149 \\
$\mathrm{C} 10 A-\mathrm{H} 11 A \cdots \mathrm{O} 32 B^{\mathrm{i}}$ & 0.99 & 2.45 & $3.251(5)$ & 138 \\
$\mathrm{C} 11 A-\mathrm{H} 13 A \cdots \mathrm{O} 52 B^{\mathrm{ii}}$ & 0.99 & 2.59 & $3.093(3)$ & 111 \\
$\mathrm{C} 2 A-\mathrm{H} 21 A \cdots \mathrm{O} 31 B^{\text {iii }}$ & 0.99 & 2.48 & $3.281(3)$ & 138
\end{tabular}

Symmetry codes: (i) $-x+3 / 2, y+1 / 2,-z+3 / 2$; (ii) $x-3 / 2,-y+3 / 2, z+1 / 2$; (iii) $-x+1 / 2, y+1 / 2,-z+3 / 2$. 